\title{
Decentralized Multiuser Detection for Time-Varying Multipath Channels
}

\author{
Tamer A. Kadous, Student Member, IEEE, and Akbar M. Sayeed, Member, IEEE
}

\begin{abstract}
Multiple-access interference (MAI) and time-varying multipath effects are the two most significant factors limiting the performance of code-division multiple-access (CDMA) systems. While multipath effects are exploited in existing CDMA systems to combat fading, they are often considered a nuisance to MAI suppression. We propose an integrated framework based on canonical multipath-Doppler coordinates that exploits channel dispersion effects for MAI suppression. The canonical coordinates are defined by a fixed basis derived from a fundamental characterization of propagation effects. The basis corresponds to uniformly spaced multipath delays and Doppler shifts of the signaling waveform that capture the essential degrees of freedom in the received signal and eliminate the need for estimating arbitrary delays and Doppler shifts. The framework builds on the notion of active coordinates that carry the desired signal energy, facilitate maximal exploitation of channel diversity, and provide minimum-complexity MAI suppression. Progressively powerful multiuser detectors are obtained by incorporating additional inactive coordinates carrying only MAI. Signal space partitioning in terms of active/inactive coordinates provides a direct handle on controlling receiver complexity to achieve a desired level of performance. System performance is analyzed for two characteristic time scales relative to the coherence time of the channel. Adaptive receiver structures are identified that are naturally amenable to blind implementations requiring knowledge of only the spreading code of the desired user.
\end{abstract}

Index Terms-Blind CDMA receivers, channel modeling, dimension reduction, interference suppression, subspace processing.

\section{INTRODUCTION}

C ODE-DIVISION multiple access (CDMA) has emerged as a promising core wireless technology for meeting the physical layer challenges of modern communication networks. Innovative signal processing is playing a key role in the design of high-performance CDMA receivers. Major signal processing challenges stem from the following three key factors that have a significant impact on CDMA system performance: channel propagation effects manifested as multipath dispersion, multipath fading, and temporal variations or Doppler effects; $m u l$ tiple-access interference (MAI); and complexity of the signal processing algorithms. Furthermore, these factors affect system performance in an interrelated fashion and have to be addressed jointly.

Paper approved by U. Mitra, the Editor for Spread Spectrum/Equalization of the IEEE Communications Society. Manuscript received August 14, 1999; revised March 28, 2000. This work was supported in part by the Wisconsin Alumni Research Foundation and by the National Science Foundation under Grant CCR-9875805. This paper was presented in part at the IEEE GLOBECOM'99, Rio de Janeiro, Brazil, December 1999.

The authors are with the Department of Electrial and Computer Engineering, University of Wisconsin-Madison, Madison, WI 53706 USA (e-mail: akbar@engr.wisc.edu).

Publisher Item Identifier S 0090-6778(00)09891-3.
For signaling waveforms of duration $T$ and bandwidth $B$, the dimension of the overall signal space is approximately ${ }^{1}$ $N_{o} \approx T B$ (see, e.g., [2]). In direct-sequence CDMA systems, $N_{o}$ is proportional to the spreading gain $N=T / T_{c}$, where $T_{c}$ is the chip duration. Centralized receivers, which have the knowledge of spreading codes of all users, represent the signal space in terms of symbol-rate sampled outputs of the matched filters for different users [3]. Decentralized receivers, which have knowledge of only the spreading code of desired user, represent the space in terms of $N$-dimensional chip-rate sampled ${ }^{2}$ outputs of the matched filter for the desired user. If the number of (strong) users $K$ is smaller than $N_{o}$, it is most advantageous to operate in the lower-dimensional subspace containing the multiuser signal. While centralized receivers directly accomplish this, decentralized receivers rely on the data itself to adaptively estimate the multiuser subspace (see, e.g., [4], [3], [5], and [6]). For desired performance of adaptive decentralized receivers in realistic time-varying scenarios, it is extremely important to map the received signal to a lower-dimensional subspace to enable reliable estimation of requisite statistics and rapid tracking. However, most existing decentralized receiver designs operate in the full $(N)$-dimensional chip-rate sampled space which can result in unacceptably poor performance in realistic time-varying scenarios (see, e.g., [7]). Multipath propagation effects distort the signal and make the problem even more challenging. While there has been considerable recent research on decentralized reception over multipath channels (see, e.g., [4] and [8]-[11], ), it falls short of jointly addressing the key issues of propagation effects, MAI suppression, and receiver complexity, primarily due to the lack of an appropriate framework relating these aspects of receiver design. In particular, there is no systematic approach for effecting a judicious complexity versus performance tradeoff.

We introduce receiver design in canonical multipath-Doppler coordinates as an integrated framework for combating timevarying multipath distortion, suppressing MAI, and managing receiver complexity. The canonical coordinates are derived from a fundamental characterization of channel propagation dynamics in terms of uniformly spaced discrete multipath delays and Doppler shifts of the signaling waveform. These waveforms capture the essential degrees of freedom in the received signal and constitute a canonical fixed basis for representing it. Consequently, processing in canonical coordinates eliminates the need for estimating arbitrary delays and Doppler shifts. While dispersion effects are often considered a nuisance to MAI

\footnotetext{
${ }^{1}$ More precisely, $N_{o}=\left(B+2 B_{d}\right)\left(T+T_{m}\right)$, where $T_{m}$ is the multipath spread and $B_{d}$ is the Doppler spread of the channel [1]. However, the terms other than $T B$ are relatively small since $T_{m} \ll T$ and $B_{d} \ll B$, typically.

${ }^{2}$ Or, oversampled outputs to cover all $N_{o} \geq N$ dimensions.
} 
suppression, canonical multipath-Doppler coordinates provide a natural partitioning of the signal space that enables exploitation of propagation effects for MAI suppression and diversity processing. A key notion in our framework is that of primary and secondary coordinates [12]. The primary (active) coordinates of a desired user depend on its multipath and Doppler spreads and define a canonical low-dimensional subspace for capturing its signal energy. The primary coordinates facilitate maximal exploitation of channel diversity [13] and minimum-complexity MAI suppression. However, additional degrees of freedom are needed in general to adequately suppress the MAI that corrupts the desired signal in the primary coordinates. These additional degrees of freedom are furnished by the secondary coordinates. The secondary coordinates may include the active coordinates of other users (centralized reception) [14], [12] or inactive coordinates of the desired user (decentralized reception) [12] that do not contain the desired signal, only the MAI. The generic receiver structure is depicted in Fig. 1(a). The signal space partitioning in terms of primary/secondary coordinates provides a systematic approach for tailoring receiver complexity to achieve a desired level of performance.

The next section develops the notion of canonical multipath-Doppler coordinates. Section III derives a decentralized minimum-mean-squared-error (MMSE) receiver structure in terms of primary and secondary coordinates. Performance analysis in Section IV guides the choice of design parameters. Examples illustrating various facets of the framework are presented in Section V. Practical issues related to channel estimation and adaptive/blind implementations are discussed in Section VI. Concluding remarks are provided in Section VII.

\section{CANONICAL MUlTiPATH-DOPPLER COORDINATES}

This section provides a brief discussion of the concept of canonical multipath-Doppler coordinates that underlies our framework [13], [12]. The complex baseband received waveform $r(t)$ for a single symbol of a single user is given by

$$
\begin{aligned}
& r(t)=s\left(t-\tau_{s}\right)+n(t) \\
& s(t)=\int_{0}^{T_{m}} \int_{-B_{d}}^{B_{d}} H(\theta, \tau) q(t-\tau) e^{j 2 \pi \theta t} d \theta d \tau
\end{aligned}
$$

where $s(t)$ is the information bearing signal, $\tau_{s}$ is the user delay, $n(t)$ is complex additive white Gaussian noise (AWGN), and $q(t)$ denotes the spread-spectrum signaling waveform of duration $T$. Channel propagation is characterized by the multipath-Doppler spreading function $H(\theta, \tau)$ that accounts for the temporal and spectral dispersion produced by the channel [15]. $T_{m}$ and $B_{d}$ denote the multipath and Doppler spreads of the channel, respectively. ${ }^{3}$

The key idea behind canonical multipath-Doppler coordinates is that the receiver "sees" only finitely many degrees of freedom in the signal due to the inherently finite duration $T$ and essentially finite bandwidth $B$ of the transmitted waveform $q(t)$ [13], [12], [15]. These essential degrees of freedom are

${ }^{3}$ We assume negligible intersymbol interference $T_{m} \ll T$, which is often the case in CDMA channels. However, the channel is frequency selective $\left(T_{m}>\right.$ $T_{c}$ ) in most cases, thereby affording multipath diversity.

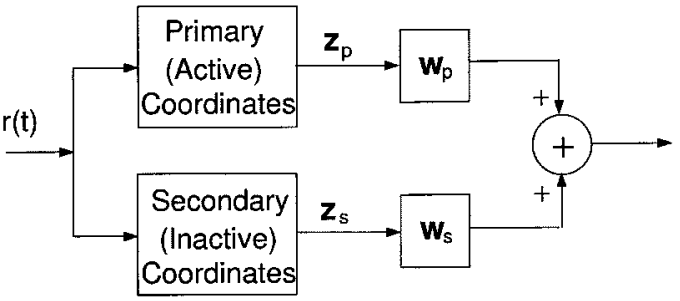

(a)

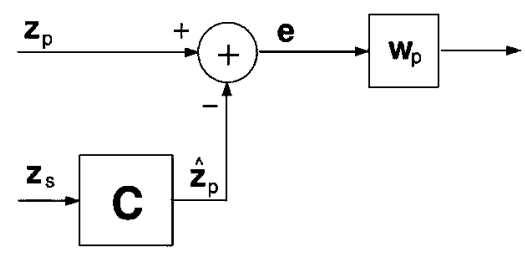

(b)

Fig. 1. (a) Generic linear receiver structure based on canonical multipathDoppler coordinates. (b) Interpretation of the MMSE receiver in (20) and (21). The matrix filter $\mathbf{C}$ suppresses MAI in the primary coordinates $\mathbf{z}_{p}$ by using the secondary coordinates $\mathbf{z}_{s}$. The filter $\mathbf{w}_{p}$ further suppresses MAI in the residual e and performs diversity combining.

captured by the following fundamental characterization [13], [12], [15], [16]:

$$
\begin{aligned}
s\left(t-\tau_{s}\right) \approx & \frac{1}{T B} \sum_{l=L_{s}}^{L_{s}+L_{a}} \sum_{m=-M_{a}}^{M_{a}} \tilde{H}\left(\frac{m}{T}, \frac{l}{B}\right) \\
& \times q\left(t-\frac{l}{B}\right) e^{j(2 \pi m t / T)} \\
= & \sum_{l=L_{s}}^{L_{s}+L_{a}} \sum_{m=-M_{a}}^{M_{a}} H_{m l} q_{m l}(t)
\end{aligned}
$$

which corresponds to a uniform sampling of the multipath-Doppler plane. $\tilde{H}(\theta, \tau)$ is a time-frequency smoothed version of $H(\theta, \tau)$ that arises due to the time- and band-limited nature of $q(t) . L_{s}=\left\lfloor\tau_{s} B\right\rfloor$ denotes the representation of the delay $\tau_{s}$. The number of degrees of freedom $\left(L_{a}+1\right)\left(2 M_{a}+1\right)$ is determined by the normalized multipath and Doppler spreads, $L_{a}=\left\lceil T_{m} B\right\rceil$ and $M_{a}=\left\lceil T B_{d}\right\rceil^{4}$ and is proportional to the products $T_{m} B_{d}$ and $T B$. We note that the Doppler components (index $m$ ) in (3) capture the temporal channel variations encountered within a symbol duration. Temporal variations over symbols are captured by the variations in the channel coefficients $\left\{H_{m l}\right\}$ over symbols.

The channel characterization (3) defines the canonical multipath-Doppler coordinates. It asserts that the received signal $s(t-$ $\left.\tau_{s}\right)$ belongs to an $\left(L_{a}+1\right)\left(2 M_{a}+1\right)$-dimensional subspace spanned by the fixed basis

$$
q_{m l}(t)=q\left(t-\frac{l}{B}\right) e^{j(2 \pi m t / T)}
$$

$l=L_{s}, L_{s}+1, \ldots, L_{s}+L_{a}, m=-M_{a}, \ldots, 0, \ldots, M_{a}$, generated by discretely delayed and Doppler-shifted versions of the spread-spectrum signaling waveform $q(t)$ [13], [12]. These basis waveforms define the active coordinates, $C_{a}=\left\{(l, m): L_{s} \leq l \leq L_{s}+L_{a},-M_{a} \leq m \leq M_{a}\right\}$

${ }^{4}\lfloor x\rfloor(\lceil x\rceil)$ denotes the largest (smallest) integer smaller (larger) than $x$. 
carrying the signal energy. Each user corresponds to unique active coordinates defined by its spreading waveform $q(t)$ and the channel spread parameters. The front-end processing for representing a received symbol in the canonical coordinates consists of projection (despreading) onto the basis waveforms of the form (4)

$$
\begin{gathered}
z_{m l}=\int r(t) q_{m l}^{*}(t) d t, \quad l=L_{1}, \ldots, L_{2} \\
m=-M_{1}, \ldots, 0, \ldots, M_{2} .
\end{gathered}
$$

In (5), $L_{1}, L_{2}, M_{1}$, and $M_{2}$ are chosen to always include the active coordinates. However, inactive coordinates, $C_{i a}=$ $\left\{(l, m) \notin C_{a}\right\}$, corresponding to canonical delays and Doppler shifts outside the channel spread, may also be used to aid in MAI suppression. Fig. 2 illustrates the notion of active/inactive coordinates. Canonical coordinates, taken together for all users and symbols of interest, along with corresponding channel coefficients, constitute sufficient statistics for demodulation-all signal processing can be performed in the canonical coordinates.

An important implication of the canonical representation (3) is that regardless of the actual physical distribution of multipath delays and Doppler shifts, virtually all information is contained in the uniformly spaced canonical coordinates [13], [12]. The main error in (3) is due to the band-limited approximation and can be made arbitrarily small by sufficient oversampling in multipath, and by including sufficiently many terms in the summation (3). In particular, for direct-sequence CDMA, $B$ is inversely related to the chip duration $T_{c}=T / N$, where $N$ is the spreading gain, and oversampling by a factor $\mathcal{O}$ corresponds to $B=\mathcal{O} / T_{c}$ in (3) and (4).

\section{RECEIVER DESIGN IN THE CANONICAL COORDINATES}

For simplicity, we illustrate multiuser reception for the case of synchronized user transmissions and binary phase-shift keying (BPSK) signaling. ${ }^{5}$ The received signal for a single symbol admits the canonical representation

$$
\begin{aligned}
r(t) & =\sum_{k=1}^{K} b_{k} s_{k}(t)+n(t) \\
s_{k}(t) & \approx \frac{1}{T B} \sum_{l=0}^{L_{k, a}} \sum_{m=-M_{k, a}}^{M_{k, a}} H_{k, m l} q_{k, m l}(t)
\end{aligned}
$$

where $b_{k}$ denotes the symbol, $\left\{q_{k, m l}(t)\right\}$ the canonical multipath-Doppler basis waveforms, and $\left\{H_{k, m l}\right\}$ the corresponding canonical channel coefficients of the $k$ th user. The above signal representation provides a natural (dictated by channel dispersion effects) a priori partitioning of the signal subspace that can be leveraged for MAI suppression. Centralized receivers represent the subspace in terms of active coordinates of all users [14]. Decentralized reception is based on an alternative representation in terms of active (primary) and inactive (secondary) coordinates of the desired user. The focus of this paper is on decentralized reception.

\footnotetext{
${ }^{5}$ Asynchronous scenarios can be treated analogously by considering twice as many interfering users and by processing a block of symbols [3]. The essential ideas presented here remain unchanged.
}

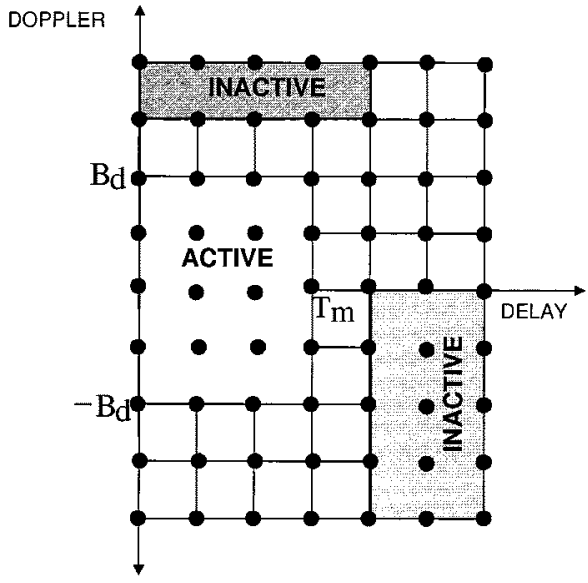

Fig. 2. A schematic illustrating active and inactive coordinates. Active coordinates correspond to multipath-Doppler basis signals that lie within the channel spread. Inactive coordinates correspond to basis functions outside the channel spread.

We illustrate the key ideas behind the framework with decentralized MMSE receiver design and begin by assuming the knowledge of the channel coefficients of the desired user. In Section VI, we discuss blind channel estimation issues and blind implementations of the proposed receivers. The overall generic receiver structure is shown in Fig. 1(a). The objective is to choose the primary and secondary filters $\mathbf{w}_{p}$ and $\mathbf{w}_{s}$ to yield an MMSE estimate of the bit $b_{1}$ of the desired user

$$
\hat{b}_{1}=\operatorname{sign}\left\{\operatorname{Re}\left(\mathbf{w}^{H} \mathbf{z}\right)\right\}=\operatorname{sign}\left\{\operatorname{Re}\left(\mathbf{w}_{p}^{H} \mathbf{z}_{p}+\mathbf{w}_{s}^{H} \mathbf{z}_{s}\right)\right\} .
$$

\section{A. Primary Coordinates-Minimal Complexity Reception}

The primary coordinates for user 1 of dimension $D_{p}=$ $\left(L_{1, a}+1\right)\left(2 M_{1, a}+1\right)$ take the form ${ }^{6}$

$$
\mathbf{z}_{p}=b_{1} \mathbf{Q}_{11} \mathbf{h}_{1}+\sum_{k=2}^{K} b_{k} \mathbf{Q}_{1 k} \mathbf{h}_{k}+\mathbf{n}_{p}=b_{1} \mathbf{g}_{1}+\mathbf{i}_{p}+\mathbf{n}_{p}
$$

where $\mathbf{Q}_{j k}$ is the $D_{p} \times D_{p}$ matrix of cross correlations between the active basis waveforms $\left\{q_{j, m l}(t):(m, l) \in C_{j, a}\right\}$ and $\left\{q_{k, m l}(t):(m, l) \in C_{k, a}\right\}$ of users $j$ and $k$, respectively, $\mathbf{h}_{k}$ denotes the $D_{p} \times 1$ vector of channel coefficients $\left\{H_{k, m l}\right\}$ of the $k$ th user, and $\mathbf{n}_{p}$ denotes $D_{p} \times 1$ Gaussian noise vector with correlation matrix $\sigma^{2} \mathbf{Q}_{11}$. The powers of different users are absorbed in the $\mathbf{h}_{k}$,'s. The first term $\mathbf{g}_{1}=\mathbf{Q}_{11} \mathbf{h}_{1}$ in (9) constitutes the signal part in $\mathbf{z}_{p}$, while the second term $\mathbf{i}_{p}$ constitutes the MAI component. Note that all the signal energy of the desired user is contained in $\mathbf{g}_{1}$. In the absence of MAI $\left(\mathbf{i}_{p}=\mathbf{0}\right)$, only the primary coordinates are needed and the optimal receiver is the generalized RAKE receiver that exploits joint multipath-Doppler diversity via maximal-ratio combining (MRC): $\mathbf{w}_{p}^{\mathrm{MRC}}=\mathbf{h}_{1}$ [13]. In the presence of MAI, the fact that the desired signal belongs to the one-dimensional subspace spanned by $\mathbf{g}_{1}$ can be exploited for suppressing $\mathbf{i}_{p}$ in (9). Given

${ }^{6}$ For simplicity, we assume the same number of primary coordinates for all users. 
$\mathbf{g}_{1}$, the MMSE receiver based only on the primary coordinates is given by

$$
\begin{aligned}
\mathbf{w}_{p}^{\mathrm{MMSE}} & =\mathbf{R}_{z_{p} z_{p}}^{-1} \mathbf{g}_{1} \\
\mathbf{R}_{z_{p} z_{p}} & =\mathrm{E}\left[\mathbf{z}_{p} \mathbf{z}_{p}^{H}\right] \\
& =\mathbf{g}_{1} \mathbf{g}_{1}^{H}+\sum_{k=2}^{K} \mathbf{Q}_{1 k} \mathrm{E}\left[\mathbf{h}_{k} \mathbf{h}_{k}^{H}\right] \mathbf{Q}_{1 k}^{H}+\sigma^{2} \mathbf{Q}_{11} .
\end{aligned}
$$

Here, $\mathbf{R}_{z_{p} z_{p}}$ is the correlation matrix of the primary coordinates. This MMSE receiver works in the low-dimensional primary coordinates to provide minimal-complexity MAI suppression while maximally exploiting the available (multipath-Doppler) diversity to combat fading.

\section{B. Primary and Secondary Coordinates-Enhanced Reception}

Secondary (inactive) coordinates $\mathbf{z}_{s}$ can be progressively incorporated into the receiver, via the lower branch in Fig. 1(a), to improve its MAI suppression capability. The secondary coordinates take the form

$$
\mathbf{z}_{s}=\sum_{k=2}^{K} b_{k} \tilde{\mathbf{Q}}_{1 k} \mathbf{h}_{k}+\mathbf{n}_{s}=\mathbf{i}_{s}+\mathbf{n}_{s}
$$

where $\tilde{\mathbf{Q}}_{1 k}$ is the $D_{s} \times D_{p}$ matrix of cross correlation between $D_{s}$ inactive basis waveforms of user 1 and the $D_{p}$ active basis waveforms of user $k$. The $D_{s} \times 1$ Gaussian noise vector $\mathbf{n}_{s}$ has correlation matrix $\sigma^{2} \tilde{\mathbf{Q}}_{s}$, where $\tilde{\mathbf{Q}}_{s}$ is the matrix of correlations between the secondary basis waveforms of user 1 . Note that a particularly attractive feature of the secondary (inactive) coordinates $\mathbf{z}_{s}$ is that they are signal free-they are only correlated with the noise and MAI component of $\mathbf{z}_{p} \cdot{ }^{7}$

The MMSE receiver operating on both the primary and secondary coordinates solves the following problem:

$$
\mathbf{w}_{O}=\arg \min _{\mathbf{w}} \mathrm{E}\left[\left|b_{1}-\mathbf{w}^{H} \mathbf{z}\right|^{2}\right]
$$

where $\mathbf{z}=\left[\begin{array}{c}\mathbf{z}_{p} \\ \mathbf{z}_{s}\end{array}\right]$ and $\mathbf{w}=\left[\begin{array}{c}\mathbf{w}_{p} \\ \mathbf{w}_{s}\end{array}\right]$. The solution to (13) is the Wiener filter and takes the form

$$
\mathbf{w}_{o}=\left[\begin{array}{l}
\mathbf{w}_{o, p} \\
\mathbf{w}_{o, s}
\end{array}\right]=\mathbf{R}_{z z}^{-1} \tilde{\mathbf{g}}_{1}
$$

where

$$
\mathbf{R}_{z z}=\mathrm{E}\left[\mathbf{z z}^{H}\right]=\left[\begin{array}{ll}
\mathbf{R}_{z_{p} z_{p}} & \mathbf{R}_{z_{p} z_{s}} \\
\mathbf{R}_{z_{s} z_{p}} & \mathbf{R}_{z_{s} z_{s}}
\end{array}\right]
$$

and $\widetilde{\mathbf{g}}_{1}=\left[\begin{array}{c}\mathbf{g}_{1} \\ \mathbf{0}\end{array}\right]$ (since the secondary coordinates are signal-free). The submatrices are given by (11) and

$$
\begin{aligned}
& \mathbf{R}_{z_{p} z_{s}}=\mathrm{E}\left[\mathbf{z}_{p} \mathbf{z}_{s}^{H}\right]=\sum_{k=2}^{K} \mathbf{Q}_{1 k} \mathrm{E}\left[\mathbf{h}_{k} \mathbf{h}_{k}^{H}\right] \tilde{\mathbf{Q}}_{1 k}^{H} \\
& \mathbf{R}_{z_{s} z_{s}}=\mathrm{E}\left[\mathbf{z}_{s} \mathbf{z}_{s}^{H}\right]=\sum_{k=2}^{K} \tilde{\mathbf{Q}}_{1 k} \mathrm{E}\left[\mathbf{h}_{k} \mathbf{h}_{k}^{H}\right] \tilde{\mathbf{Q}}_{1 k}^{H}+\sigma^{2} \tilde{\mathbf{Q}}_{s} .
\end{aligned}
$$

${ }^{7}$ Note that in (12) we are assuming that $\tilde{\mathbf{Q}}_{11} \approx \mathbf{0}$; that is, the active and inactive basis waveforms are roughly orthogonal. This assumption is based on the autocorrelation properties of spreading codes. However, our approach can be readily extended to account for nonzero correlations as well. In particular, we may use a linearly transformed version of the inactive coordinates that lie in the orthogonal component of the active subspace.
By using the block matrix inversion formula [17], we can expand $\mathbf{R}_{z z}^{-1}$ as

$$
\mathbf{R}_{z z}^{-1}=\left[\begin{array}{cc}
\mathbf{R}_{\mathrm{ee}}^{-1} & -\mathbf{R}_{\mathrm{ee}}^{-1} \mathbf{C}^{H} \\
-\mathbf{C R}_{\mathrm{ee}}^{-1} & \left(\mathbf{R}_{z_{s} z_{s}}-\mathbf{R}_{z_{s} z_{p}} \mathbf{R}_{z_{p} z_{p}}^{-1} \mathbf{R}_{z_{p} z_{s}}\right)^{-1}
\end{array}\right]
$$

where

$$
\begin{aligned}
\mathbf{C} & =\mathbf{R}_{z_{s} z_{s}}^{-1} \mathbf{R}_{z_{s} z_{p}}, \quad \mathbf{e}=\mathbf{z}_{p}-\mathbf{C}^{H} \mathbf{z}_{s} \\
\mathbf{R}_{\mathrm{ee}} & =\mathrm{E}\left[\mathbf{e e}^{H}\right]=\mathbf{R}_{z_{p} z_{p}}-\mathbf{R}_{z_{p} z_{s}} \mathbf{R}_{z_{s} z_{s}}^{-1} \mathbf{R}_{z_{s} z_{p}} .
\end{aligned}
$$

Using (14) and (17), we can explicitly characterize $\mathbf{w}_{o, p}$ and $\mathbf{w}_{o, s}$ as

$$
\begin{aligned}
& \mathbf{w}_{o, p}=\mathbf{R}_{\mathrm{ee}}^{-1} \mathbf{g}_{1} \\
& \mathbf{w}_{o, s}=-\mathbf{C w}_{o, p}=-\mathbf{C R}_{\mathrm{ee}}^{-1} \mathbf{g}_{1} .
\end{aligned}
$$

The solution for the primary and secondary filters in (20) and (21) has an intuitively appealing interpretation as illustrated in Fig. 1(b). Conditioned on a fixed value of the $\mathbf{g}_{1}=\mathbf{Q}_{11} \mathbf{h}_{1}$, the matrix $\mathbf{C}$ is the linear MMSE estimator of $\mathbf{z}_{p}$ from $\mathbf{z}_{s}\left(\hat{\mathbf{z}}_{p}=\right.$ $\mathbf{C}^{H} \mathbf{z}_{s}$ ) and $\mathbf{R}_{\mathrm{ee}}$ is the covariance matrix of the corresponding estimation error $\mathbf{e}$. Thus, the filter $\mathbf{C}$ optimally exploits the secondary coordinates $\mathbf{z}_{s}$ to suppress MAI in $\mathbf{z}_{p}$ (since $\mathbf{z}_{s}$ is uncorrelated with the signal component $\mathbf{g}_{1}$ in $\mathbf{z}_{p}$ ). The filter $\mathbf{w}_{o, p}$ then optimally suppresses the MAI remaining in the residual error $\mathbf{e}$ by forming an MMSE estimate of $\hat{b}_{1}$ from e (compare with the solution in (11) based on the primary coordinates only). The number of secondary coordinates $D_{s}$ can vary between zero (only primary coordinates) and $N_{o}-D_{p}$ (covering the entire signal space). As we will see, depending on the number of dominant interfering users, near-optimal performance can be achieved with significantly low-dimensional $\left(D_{p}+D_{s} \ll N\right)$ processing.

We note that most of the decentralized receivers proposed in the literature are based on chip-rate sampled processing. The received signal is sampled every chip duration and the resultant $N$-dimensional vector is processed by an $N$-tap filter, which may be chosen based on the MMSE criterion. In practice, the $N$-tap MMSE receiver has to be implemented adaptively. For large $N$, there are too many degrees of freedom to allow reliable estimation and tracking of the MMSE filter taps. Thus, it is necessary to develop MMSE detectors with fewer number of taps (degrees of freedom). Ad hoc lower-dimensional representations for the filter are often considered (see, for example, [18]). Other approaches based on subspace tracking are presented in [19] and, [9]. In our framework, the natural signal space partitioning in terms of active/inactive coordinates provides a systematic approach to controlling receiver complexity. The detector operates in an $N_{\text {tot }}=D_{s}+D_{p} \leq N$-dimensional subspace. The complexity of the receiver can be progressively increased, by increasing $D_{s}$, to achieve a desired level of performance.

We also note that the proposed receiver only requires knowledge of $\mathbf{g}_{1}=\mathbf{Q}_{11} \mathbf{h}_{1}$ and $\mathbf{R}_{z z} \cdot \mathbf{Q}_{11}$ only depends on the spreading code of the desired user and $\mathbf{R}_{z z}$ can be readily estimated from data. This solution assumes the knowledge of $\mathbf{h}_{1}$ of the desired user. In Section VI-B, we will see that the structure of $\mathbf{e}$ can be exploited for blindly estimating $\mathbf{h}_{1}$.

\section{Performance AnALysis}

In this section, we assess the performance of the proposed MMSE receiver structure under varying conditions. Let $T_{\text {obs }}$ 
be the time scale over which the performance of the receiver is assessed. Our analysis is based on two distinct time scales depending on how $T_{\mathrm{obs}}$ compares $T_{\mathrm{coh}, d}$, the coherence time of the channel seen by the desired user. Essentially, $T_{\mathrm{coh}, d}$ is the time duration over which the channel coefficients $\mathbf{h}_{1}$ (and hence $\mathbf{g}_{1}$ ) remain approximately constant. The coherence time is roughly equal to the reciprocal of the channel Doppler spread $\left(1 / B_{d}\right)$ [1]. For $T_{\mathrm{obs}}<T_{\mathrm{coh}, d}$, the desired user's signal exhibits a fixed direction determined by $\mathbf{g}_{1}$ and the channel is effectively equivalent to an AWGN channel. Thus, the probability of error $\left(P_{e}\right)$ is governed by the instantaneous signal-to-interference-plus-noise ratio (SINR) $\operatorname{SINR}\left(\mathbf{g}_{1}\right)$ [20]. ${ }^{8}$ Over time scales significantly longer than $T_{\mathrm{coh}, d}$, i.e., $T_{\mathrm{obs}} \gg T_{\mathrm{coh}, d}$, the desired user's signal can exhibit up to $D_{p}=\left(L_{1, a}+1\right)\left(2 M_{1, a}+1\right)$ degrees of freedom and the average $P_{e}$ is computed by averaging $\operatorname{SINR}\left(\mathbf{g}_{1}\right)$ over the statistics of $\mathbf{g}_{1}$. We analyze the receiver over both time scales and also investigate measures of near-far resistance [3] that are appropriate in the two scenarios.

Another factor that affects system performance is the degrees of freedom exhibited by the interference. This depends on how $T_{\text {obs }}$ compares to the coherence time of the interference $T_{\mathrm{coh}, i} \cdot{ }^{9}$ If $T_{\mathrm{obs}}<T_{\mathrm{coh}, i}$, the MAI exhibits up to $N_{i}=(K-1)$ degrees of freedom in the full-dimensional space since each interfering user exhibits a fixed direction within its active subspace $\left[\mathrm{E}\left[\mathbf{h}_{k} \mathbf{h}_{k}^{H}\right] \approx \mathbf{h}_{k} \mathbf{h}_{k}^{H}\right.$ in (11), (15), and (16)]. On the other hand, if $T_{\text {obs }} \gg T_{\mathrm{coh}, i}$, the MAI can exhibit up to $N_{i}=(K-1) D_{p}$ degrees of freedom, since each interfering user can exhibit up to $D_{p}$ directions in its active subspace due to the time variations in the channel coefficients. ${ }^{10}$ Clearly, in the detector subspace, spanned by the active and inactive basis vectors, the MAI cannot exhibit more than $N_{\text {tot }}=D_{p}+D_{s}$ degrees of freedom. However, unless $N_{\text {tot }} \geq N_{i}+1$, the receiver will not be near-far resistant. As long as $N_{i}+1<N$, we can choose $D_{s}$ to ensure $N_{\text {tot }} \geq N_{i}+1$. Our analysis will clearly show the dependence of performance on $N_{i}$ and $N_{\text {tot }}$.

To facilitate analysis, we first derive alternate expressions for the optimum solution. Let $\mathbf{y}=\mathbf{z}-\tilde{\mathbf{g}}=\mathbf{i}+\mathbf{n}$ denote the signal-free component of the canonical coordinates

$$
\mathbf{y}=\left[\begin{array}{l}
\mathbf{y}_{p} \\
\mathbf{y}_{s}
\end{array}\right]=\left[\begin{array}{l}
\mathbf{z}_{p} \\
\mathbf{z}_{s}
\end{array}\right]-\left[\begin{array}{c}
\mathbf{g}_{1} \\
\mathbf{0}
\end{array}\right]=\left[\begin{array}{l}
\mathbf{i}_{p} \\
\mathbf{i}_{s}
\end{array}\right]+\left[\begin{array}{l}
\mathbf{n}_{p} \\
\mathbf{n}_{s}
\end{array}\right]
$$

The correlation matrix of $y$ is of the form

$$
\begin{aligned}
\mathbf{R}_{y y} & =\mathbf{R}_{z z}-\tilde{\mathbf{g}}_{1} \tilde{\mathbf{g}}_{1}^{H}=\mathbf{R}_{i i}+\mathbf{R}_{n n} \\
& =\left[\begin{array}{ll}
\mathbf{R}_{i_{p} i_{p}} & \mathbf{R}_{i_{p} i_{s}} \\
\mathbf{R}_{i_{s} i_{p}} & \mathbf{R}_{i_{s} i_{s}}
\end{array}\right]+\sigma^{2}\left[\begin{array}{cc}
\mathbf{Q}_{11} & \mathbf{0} \\
\mathbf{0} & \tilde{\mathbf{Q}}_{s}
\end{array}\right] .
\end{aligned}
$$

${ }^{8}$ Note that SINR is really a function of all $\mathbf{h}_{k}$ 's. However, for sufficiently effective MAI suppression, the residual MAI can be lumped to Gaussain noise [20].

${ }^{9}$ For simplicity, we assume that all interfering users experience the same coherence time $T_{\mathrm{col}, i}$ ( which may be different from $T_{\mathrm{col}_{1}, d}$ ).

${ }^{10}$ Note that $\operatorname{rank}\left(E\left[\mathbf{h}_{k} \mathbf{h}_{k}^{H}\right]\right) \leq D_{p}$
Without loss of generality assume that $\mathbf{R}_{n n}=\sigma^{2} \mathbf{I}$; that is, $\mathbf{Q}_{11}=\mathbf{I}$ and $\tilde{\mathbf{Q}}_{s}=\mathbf{I} .{ }^{11}$ Let

$$
\begin{aligned}
\mathbf{R}_{i i} & =\left[\begin{array}{ll}
\mathbf{V}_{I} & \mathbf{V}_{\perp}
\end{array}\right]\left[\begin{array}{cc}
\boldsymbol{\Lambda} & \mathbf{0} \\
\mathbf{0} & \mathbf{0}
\end{array}\right]\left[\begin{array}{l}
\mathbf{V}_{I}^{H} \\
\mathbf{V}_{\perp}^{H}
\end{array}\right] \\
& =\left[\begin{array}{ll}
\mathbf{V}_{I, p} & \mathbf{V}_{\perp, p} \\
\mathbf{V}_{I, s} & \mathbf{V}_{\perp, s}
\end{array}\right]\left[\begin{array}{cc}
\boldsymbol{\Lambda} & \mathbf{0} \\
\mathbf{0} & \mathbf{0}
\end{array}\right]\left[\begin{array}{ll}
\mathbf{V}_{I, p}^{H} & \mathbf{V}_{I, s}^{H} \\
\mathbf{V}_{\perp, p}^{H} & \mathbf{V}_{\perp, s}^{H}
\end{array}\right]
\end{aligned}
$$

be the eigendecomposition of $\mathbf{R}_{i i}$, where $\mathbf{V}_{I}$ contains the eigenvectors corresponding to nonzero eigenvalues ( $\boldsymbol{\Lambda}$ is the diagonal matrix of nonzero eigenvalues) and $\mathbf{V}_{\perp}$ contains the eigenvectors corresponding to the zero eigenvalues. The second equality further partitions $\mathbf{V}_{I}$ and $\mathbf{V}_{\perp}$ into primary and secondary coordinates. Let $\mathcal{S}_{I}=\operatorname{span}\left(\mathbf{V}_{I}\right)$ denote the interference subspace and $\mathcal{S}_{\perp}=\operatorname{span}\left(\mathbf{V}_{\perp}\right)$ denote the orthogonal complement of $\mathcal{S}_{I}$. Note that $\operatorname{dim}\left(\mathcal{S}_{I}\right)=\min \left(N_{i}, N_{\text {tot }}\right)$ and $\operatorname{dim}\left(\mathcal{S}_{\perp}\right)=N_{\text {tot }}$ $-\operatorname{dim}\left(\mathcal{S}_{I}\right)$.

$\mathbf{R}_{y y}$ admits the eigendecomposition

$$
\mathbf{R}_{y y}=\mathbf{V}_{I} \tilde{\mathbf{\Lambda}} \mathbf{V}_{I}^{H}+\sigma^{2} \mathbf{V}_{\perp} \mathbf{V}_{\perp}^{H}
$$

where $\tilde{\boldsymbol{\Lambda}}=\boldsymbol{\Lambda}+\sigma^{2} \mathbf{I}$. Using the block inversion formula (17) and (25), we get

$$
\begin{aligned}
\mathbf{R}_{y y}^{-1}= & {\left[\begin{array}{cc}
\mathbf{R}_{\epsilon \epsilon}^{-1} & -\mathbf{R}_{\epsilon \epsilon}^{-1} \mathbf{C}^{H} \\
-\mathbf{C R}_{\epsilon \epsilon}^{-1} & \mathbf{D}
\end{array}\right] } \\
= & \mathbf{V}_{I} \tilde{\mathbf{\Lambda}}^{-1} \mathbf{V}_{I}^{H}+\frac{1}{\sigma^{2}} \mathbf{V}_{\perp} \mathbf{V}_{\perp}^{H} \\
= & {\left[\begin{array}{cc}
\mathbf{V}_{I, p} \tilde{\boldsymbol{\Lambda}}^{-1} \mathbf{V}_{I, p}^{H} & \mathbf{V}_{I, p} \tilde{\mathbf{\Lambda}}^{-1} \mathbf{V}_{I, s}^{H} \\
\mathbf{V}_{I, s} \tilde{\boldsymbol{\Lambda}}^{-1} \mathbf{V}_{I, p}^{H} & \mathbf{V}_{I, s} \tilde{\boldsymbol{\Lambda}}^{-1} \mathbf{V}_{I, s}^{H}
\end{array}\right] } \\
& +\frac{1}{\sigma^{2}}\left[\begin{array}{ll}
\mathbf{V}_{\perp, p} \mathbf{V}_{\perp, p}^{H} & \mathbf{V}_{\perp, p} \mathbf{V}_{\perp, s}^{H} \\
\mathbf{V}_{\perp, s} \mathbf{V}_{\perp, p}^{H} & \mathbf{V}_{\perp, s} \mathbf{V}_{\perp, s}^{H}
\end{array}\right]
\end{aligned}
$$

where $\epsilon=\mathbf{e}-\mathbf{g}_{1}$ denotes the signal-free component of the residual estimation error $\mathbf{e}\left(\mathbf{R}_{\epsilon \epsilon}=\mathbf{R}_{\mathrm{ee}}-\mathbf{g}_{1} \mathbf{g}_{1}^{H}\right)$ and $\mathbf{D}=$ $\left(\mathbf{R}_{y_{s} y_{s}}-\mathbf{R}_{y_{s} y_{p}} \mathbf{R}_{y_{p} y_{p}}^{-1} \mathbf{R}_{y_{p} y_{s}}\right)^{-1}$. Based on the preceding development, the optimum filter solution admits the following representations:

$$
\begin{aligned}
\mathbf{w}_{o}= & \mathbf{R}_{z z}^{-1} \tilde{\mathbf{g}}_{1}=\frac{\mathbf{R}_{y y}^{-1} \tilde{\mathbf{g}}_{1}}{1+\tilde{\mathbf{g}}_{1}^{H} \mathbf{R}_{y y}^{-1} \tilde{\mathbf{g}}_{1}} \\
= & {\left[\begin{array}{c}
\mathbf{w}_{o, p} \\
\mathbf{w}_{o, s}
\end{array}\right]=\frac{1}{1+\mathbf{g}_{1}^{H} \mathbf{R}_{\epsilon \epsilon}^{-1} \mathbf{g}_{1}}\left[\begin{array}{c}
\mathbf{R}_{\epsilon \epsilon}^{-1} \mathbf{g}_{1} \\
-\mathbf{C R}_{\epsilon \epsilon}^{-1} \mathbf{g}_{1}
\end{array}\right] } \\
= & \frac{1}{\sigma^{2}+\mathbf{g}_{1}^{H}\left(\sigma^{2} \mathbf{V}_{I, p} \tilde{\boldsymbol{\Lambda}}^{-1} \mathbf{V}_{I, p}^{H}+\mathbf{V}_{\perp, p} \mathbf{V}_{\perp, p}^{H}\right) \mathbf{g}_{1}} \\
& \times\left[\begin{array}{c}
\left(\sigma^{2} \mathbf{V}_{I, p} \tilde{\boldsymbol{\Lambda}}^{-1} \mathbf{V}_{I, p}^{H}+\mathbf{V}_{\perp, p} \mathbf{V}_{\perp, p}^{H}\right) \mathbf{g}_{1} \\
\left(\sigma^{2} \mathbf{V}_{I, s} \tilde{\mathbf{\Lambda}}^{-1} \mathbf{V}_{I, p}^{H}+\mathbf{V}_{\perp, s} \mathbf{V}_{\perp, p}^{H}\right) \mathbf{g}_{1}
\end{array}\right]
\end{aligned}
$$

where the first equality follows from (23) and the matrix inversion lemma [21], the second one from (26), and the last one

\footnotetext{
${ }^{11}$ Otherwise, we can always prewhiten the canonical coordinates: $\mathbf{z} \rightarrow \mathbf{R}_{n n}^{-1 / 2} \mathbf{z}$, since $\mathbf{Q}_{11}$ and $\tilde{\mathbf{Q}}_{s}$ are known at the receiver.
} 
from (27). Finally, we note that $\tilde{\mathrm{g}}$ admits the following unique orthogonal decomposition:

$$
\tilde{\mathrm{g}}=\tilde{\mathrm{g}}_{I}+\tilde{\mathrm{g}}_{\perp}
$$

where

and

$$
\tilde{\mathbf{g}}_{I}=\mathbf{V}_{I} \mathbf{V}_{I}^{H} \widetilde{\mathbf{g}}=\left[\begin{array}{c}
\mathbf{V}_{I, p} \\
\mathbf{V}_{I, s}
\end{array}\right] \mathbf{V}_{I, p}^{H} \mathbf{g}_{1}
$$

$$
\tilde{\mathbf{g}}_{\perp}=\mathbf{V}_{\perp} \mathbf{V}_{\perp}^{H} \tilde{\mathbf{g}}=\left[\begin{array}{c}
\mathbf{V}_{\perp, p} \\
\mathbf{V}_{\perp, s}
\end{array}\right] \mathbf{V}_{\perp, p}^{H} \mathbf{g}_{1} .
$$

In particular, $\mathbf{g}_{1}$ admits the following (nonorthogonal) decomposition:

$$
\mathbf{g}_{1}=\mathbf{g}_{1, I}+\mathbf{g}_{1, \perp}=\mathbf{V}_{I, p} \mathbf{V}_{I, p}^{H} \mathbf{g}_{1}+\mathbf{V}_{\perp, p} \mathbf{V}_{\perp, p}^{H} \mathbf{g}_{1} .
$$

\section{A. Limiting Solution at High Signal-to-Noise Ratio (SNR)}

From (30) and (32) it follows that

$$
\begin{aligned}
\lim _{\sigma^{2} \rightarrow 0} \mathbf{w}_{o} & =\lim _{\sigma^{2} \rightarrow 0}\left[\begin{array}{c}
\mathbf{w}_{o, p} \\
\mathbf{w}_{o, s}
\end{array}\right] \\
& =\frac{1}{\mathbf{g}_{1}^{H} \mathbf{V}_{\perp, p} \mathbf{V}_{\perp, p}^{H} \mathbf{g}_{1}}\left[\begin{array}{c}
\mathbf{V}_{\perp, p} \mathbf{V}_{\perp, p}^{H} \mathbf{g}_{1} \\
\mathbf{V}_{\perp, s} \mathbf{V}_{\perp, p}^{H} \mathbf{g}_{1}
\end{array}\right] \\
& =\frac{\tilde{\mathbf{g}}_{\perp}}{\left\|\tilde{\mathbf{g}}_{\perp}\right\|^{2}} .
\end{aligned}
$$

Recall that $\mathcal{S}_{\perp}$ is nonempty as long as $N_{\text {tot }} \geq N_{i}+1$ (the total number of coordinates is greater than or equal to the dimension of the signal-plus-interference subspace). It follows from (33) that in the limit of high SNR $\left(\sigma^{2} \rightarrow 0\right)$, as long as $N_{\text {tot }} \geq N_{i}+$ 1 , the optimum filter solution converges to a unit vector in the direction of $\tilde{\mathbf{g}}_{\perp} \in \mathcal{S}_{\perp} .{ }^{12}$ That is, MAI is completely suppressed in the output of the MMSE receiver in the limit of high SNR if $N_{\text {tot }} \geq N_{i}+1$. Thus, in the limit of high SNR, MAI with up to $N_{i}=D_{p}-1$ degrees of freedom can be suppressed with only the primary coordinates. This means that in a sufficiently underloaded system, the primary coordinates [the upper branch in Fig. 1(a)] are adequate for required MAI suppression. On the other hand, the augmented receiver based on both primary and secondary coordinates is capable of suppressing MAI with up to $N_{i}=D_{p}+D_{s}-1$ dominant degrees of freedom. Thus, by progressively adding more secondary coordinates, we can suppress a larger number of interfering users.

\section{B. Probability of Error}

As mentioned earlier, if $T_{\mathrm{obs}}<T_{\mathrm{coh}, d}$, receiver performance is fairly accurately characterized by the SINR. The SINR is defined as the ratio of the desired signal power to the interference and noise power at the output of the receiver, and in our formulation it takes the form $\operatorname{SINR}\left(\mathbf{g}_{1}\right)=$ $\left(\left|\mathbf{w}_{o, p}^{H} \mathbf{g}_{1}\right|^{2} /\left(\mathrm{E}\left[\left|\mathbf{w}_{o}^{H} \mathbf{z}\right|^{2}\right]-\left|\mathbf{w}_{o, p}^{H} \mathbf{g}_{1}\right|^{2}\right)\right)$. Using the various forms of the solution in (28)-(30), the SINR can be expressed as

$$
\begin{aligned}
\operatorname{SINR}\left(\mathbf{g}_{1}\right) & =\frac{\tilde{\mathbf{g}}^{H} \mathbf{R}_{z z}^{-1} \tilde{\mathbf{g}}}{1-\tilde{\mathbf{g}}^{H} \mathbf{R}_{z z}^{-1} \tilde{\mathbf{g}}}=\frac{\mathbf{g}_{1}^{H} \mathbf{R}_{\mathrm{ee}}^{-1} \mathbf{g}_{1}}{1-\mathbf{g}_{1}^{H} \mathbf{R}_{\mathrm{ce}}^{-1} \mathbf{g}_{1}} \\
& =\tilde{\mathbf{g}}^{H} \mathbf{R}_{y y}^{-1} \tilde{\mathbf{g}}=\mathbf{g}_{1}^{H} \mathbf{R}_{\epsilon \epsilon}^{-1} \mathbf{g}_{1}
\end{aligned}
$$

${ }^{12}$ This result parallels a similar result in [18] for the AWGN channel.

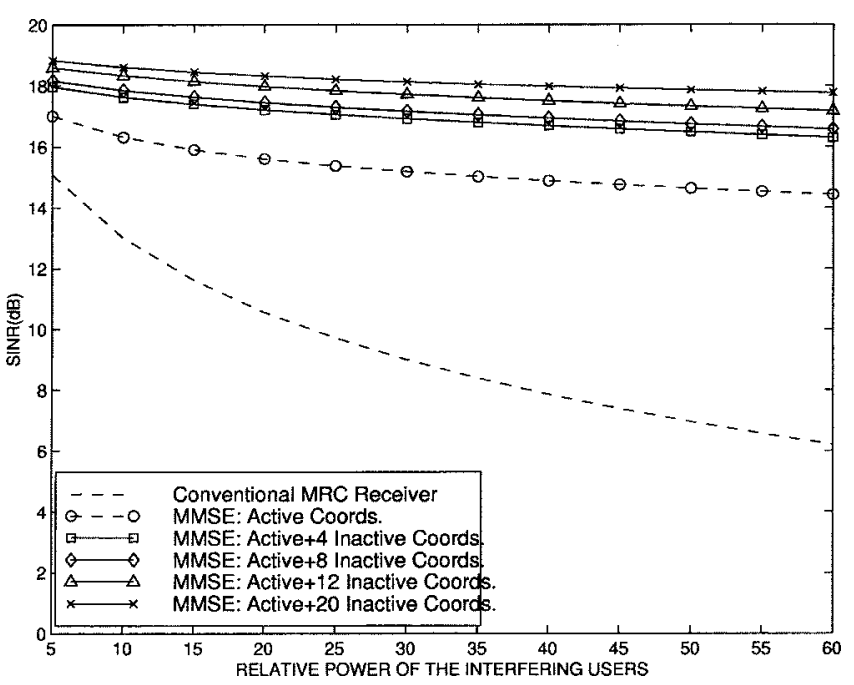

(a)

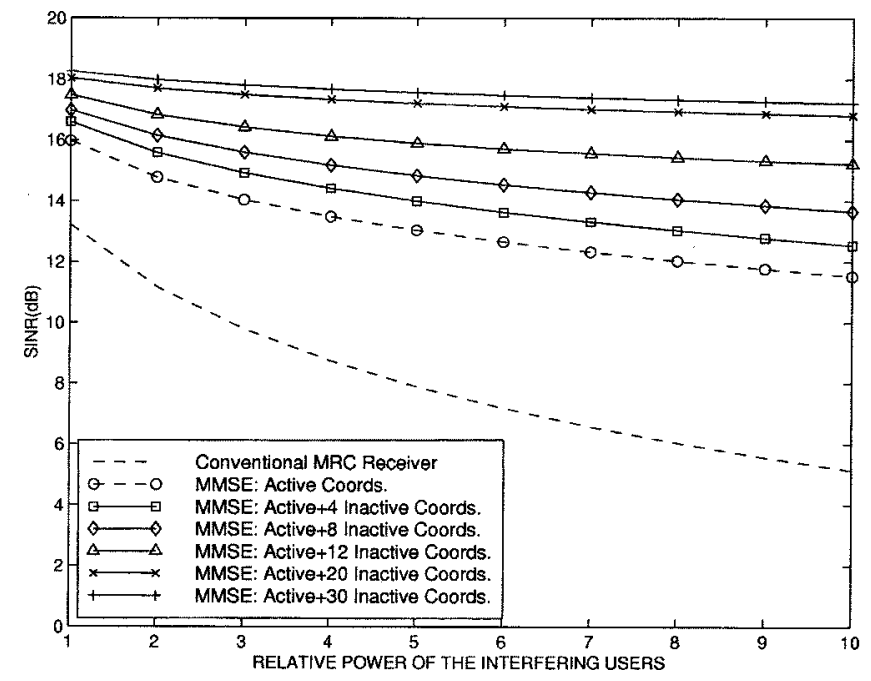

(b)

Fig. 3. Receiver SINR versus relative power of each interfering user for different number of secondary coordinates $\left(D_{s}\right)$. Long times scales with respect to interference coherence time $\left(T_{\mathrm{obs}} \gg T_{\mathrm{col}, i}\right)$. (a) One interfering user $\left(N_{i}=9\right)$. (b) Three interfering users $\left(N_{i}=27\right)$. Notice the saturation of SINR as $N_{\text {tot }}=D_{p}+D_{s}=9+D_{s}$ increases beyond $N_{i}+1$.

$$
=\mathbf{g}_{1}^{H}\left(\mathbf{V}_{I, p} \tilde{\mathbf{\Lambda}}^{-1} \mathbf{V}_{I, p}^{H}+\frac{1}{\sigma^{2}} \mathbf{V}_{\perp, p} \mathbf{V}_{\perp, p}^{H}\right) \mathbf{g}_{1} .
$$

Note that SINR depends on the particular value of $\mathbf{g}_{1}$. Drawing on the discussion in [20], we argue that for sufficiently large $N_{\text {tot }}=D_{p}+D_{s}, P_{e}$ is fairly accurately given by

$$
P_{e}\left(\mathbf{g}_{1}\right)=Q\left(\sqrt{2 \operatorname{SINR}\left(\mathbf{g}_{1}\right)}\right)
$$

over short time scales, where

$$
Q(x)=\int_{x}^{\infty} \frac{1}{\sqrt{2 \pi}} e^{-x^{2} / 2} d x .
$$

We note that the SINR is a nondecreasing function of the total number of coordinates. As we saw in Section IV-A, in the limit of high SNR only $N_{\text {tot }}=N_{i}+1$ coordinates are necessary for suppressing $N_{i}$ interferers. As we see in Section V, the SINR shows relatively small improvement as $N_{\text {tot }}$ is increased beyond $N_{i}+1$ by increasing $D_{s}$ (see Figs. 3 and 4 ). 


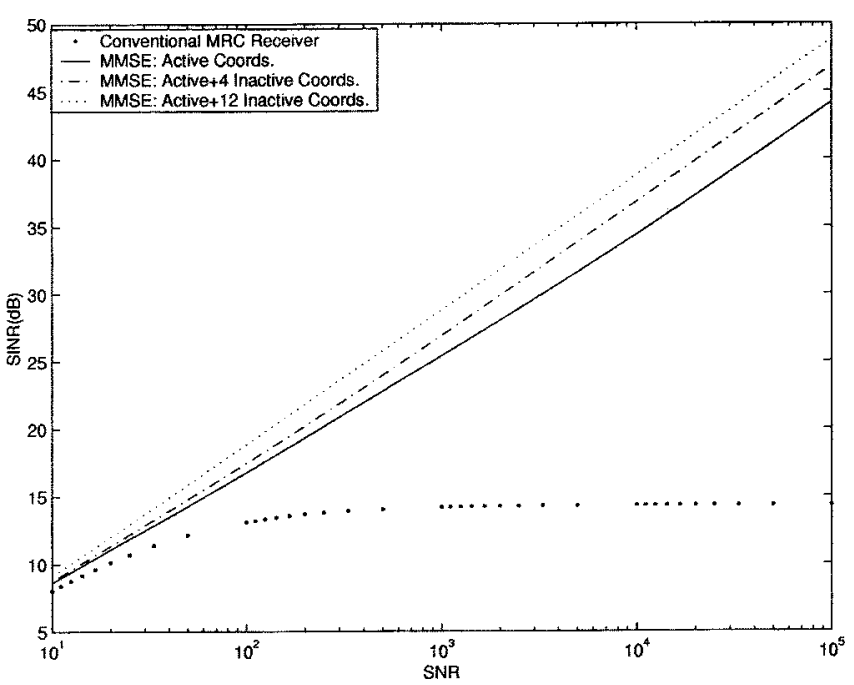

(a)

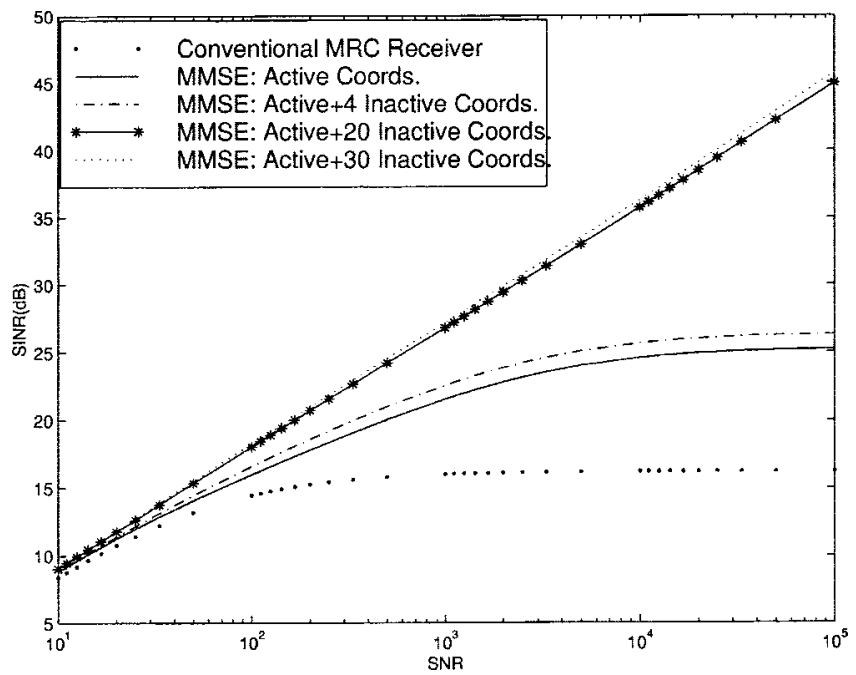

(b)

Fig. 4. Receiver SINR as a function of SNR of desired user for different values of $D_{s}$. Three interfering users with the same power as the desired user. (a) $T_{\mathrm{obs}}<T_{\mathrm{col}, i}\left(N_{i}=3\right)$. (b) $T_{\mathrm{obs}} \gg T_{\mathrm{col}, i}\left(N_{i}=27\right)$. Note that the receiver moves from an MAI-limited regime to a noise-limited regime as $D_{s}$ increases beyond $N_{i}+1-D_{p}$ [ -5 for (a) and 19 for (b)]. SINR saturates in the MAI-limited regime but increases linearly with SNR in the noise-limited regime.

To compute the $P_{e}$ over time scales much longer than $T_{\text {coh, } d}$ (i.e., $T_{\text {obs }} \gg T_{\text {coh }, d}$ ), we need to average the conditional expression in (37) over the distribution of $\mathbf{g}_{1} \cdot{ }^{13}$ Since $\mathbf{g}_{1}$ is modeled as Gaussian, the SINR expression in (35) can be alternatively expressed as $\operatorname{SINR}\left(\mathbf{g}_{1}\right)=\sum_{l=1}^{D_{p}} \mu_{l} \gamma_{l}$, where $\mu_{l}, l=$ $1,2, \ldots, D_{p}$, are the eigenvalues of $\mathbf{R}_{\epsilon \epsilon}^{-1} \mathbf{R}_{g_{1}}, \mathbf{R}_{g_{1}}=\mathrm{E}\left[\mathbf{g}_{1} \mathbf{g}_{1}^{H}\right]$ $=\mathbf{Q}_{11} \mathrm{E}\left[\mathbf{h}_{1} \mathbf{h}_{1}^{H}\right] \mathbf{Q}_{11}$, and $\gamma_{l}, l=1,2, \ldots, D_{p}$, are independent $\chi^{2}$ random variables with two degrees of freedom and $\mathrm{E}\left[\left|\gamma_{l}\right|^{2}\right]=1$. Assuming distinct eigenvalues $\left\{\mu_{l}\right\}$, the $P_{e}$ can be computed as [1]

$$
P_{e}=\mathrm{E}\left[P_{e}\left(\mathbf{g}_{1}\right)\right]=\sum_{l=1}^{D_{p}} \frac{\pi_{l}}{2}\left[1-\sqrt{\frac{\mu_{l}}{1+\mu_{l}}}\right]
$$

\footnotetext{
${ }^{13}$ Strictly speaking, we need to average over the distributions of all $\mathbf{h}_{k}$ 's. However, along the lines of [20], we are treating the MAI collectively as Gaussian noise. Fig. 6 supports this approximation.
}

where

$$
\pi_{l}=\prod_{i=1, i \neq l}^{D_{p}} \frac{\mu_{l}}{\mu_{l}-\mu_{i}}
$$

\section{Near-Far Resistance}

Over time scales within $T_{\text {coh, }}$, the proposed multiuser MMSE receivers are "near-far resistant" in the sense that the $\operatorname{SINR}\left(\mathbf{g}_{1}\right)>0$ even in the limit of infinite interference power as long as $\tilde{\mathbf{g}}_{\perp} \neq \mathbf{0}$, as evident from (30) and (36)

and

$$
\lim _{\Lambda_{j j} \rightarrow \infty} \mathbf{w}_{O}=\frac{\tilde{\mathbf{g}}_{\perp}}{\sigma^{2}+\left\|\tilde{\mathbf{g}}_{\perp}\right\|^{2}}
$$

$$
\lim _{\boldsymbol{\Lambda}_{j j} \rightarrow \infty} \operatorname{SINR}\left(\mathbf{g}_{1}\right)=\frac{\left\|\tilde{\mathbf{g}}_{\perp}\right\|^{2}}{\sigma^{2}} \quad\left(N_{\text {tot }} \geq N_{i}+1\right)
$$

where $\boldsymbol{\Lambda}_{j j}$ denote the nonzero eigenvalues of $\mathbf{R}_{i i}$ in (24). We note that in the limit of strong interference (similar to $\sigma^{2} \rightarrow$ 0 ) the optimal filter aligns itself orthogonal to the interference subspace and thus completely suppresses the MAI.

We also investigate the notion of near-far resistance defined in [3] and [22] based on the asymptotic relative efficiency (ARE) of the receiver. The ARE of the multiuser receiver compares its asymptotic performance (as $\sigma^{2} \rightarrow 0$ ) in the presence of MAI relative to its performance in the absence of MAI. For time scales within $T_{\text {coh, } d}$, the ARE is defined as

$$
\nu\left(\mathbf{g}_{1}\right)=\sup \left\{0 \leq r \leq 1: \lim _{\sigma^{2} \rightarrow 0} \frac{P_{e}\left(\mathbf{g}_{1}, \sigma\right)}{Q\left(\sqrt{\frac{2 r\left\|\mathbf{g}_{1}\right\|^{2}}{\sigma^{2}}}\right)}=0\right\}
$$

where $P_{e}\left(\mathbf{g}_{1}, \sigma\right)$ is the error probability of the receiver in the presence of MAI as a function of $\sigma$ [implicit in (37)], and $Q\left(\sqrt{2 r\left\|\mathrm{~g}_{1}\right\|^{2} / \sigma}\right)$ captures the $P_{e}$ of the corresponding single-user MRC receiver. The near-far resistance is defined as [3], [22] $\eta\left(\mathbf{g}_{1}\right)=\inf _{\boldsymbol{\Lambda}_{j j}} \nu\left(\mathbf{g}_{1}\right)$. From (36) and (37), we note that as $\sigma^{2} \rightarrow 0, P_{e}\left(\mathbf{g}_{1}, \sigma\right) \approx Q\left(\sqrt{\left.2\left\|\tilde{\mathbf{g}}_{\perp}\right\|\right|^{2} / \sigma^{2}}\right)$ from which it follows that $\nu\left(\mathbf{g}_{1}\right)=\left\|\tilde{\mathbf{g}}_{\perp}\right\|^{2} /\left\|\mathbf{g}_{1}\right\|^{2}$. Since $\nu\left(\mathbf{g}_{1}\right)$ is independent of the interference power, it follows that ${ }^{14}$

$$
\eta\left(\mathbf{g}_{1}\right)=\nu\left(\mathbf{g}_{1}\right)=\frac{\left\|\tilde{\mathbf{g}}_{\perp}\right\|^{2}}{\left\|\mathbf{g}_{1}\right\|^{2}}=\frac{\left\|\tilde{\mathbf{g}}_{\perp}\right\|^{2}}{\|\tilde{\mathbf{g}}\|^{2}} .
$$

It is clear that $\eta\left(\mathbf{g}_{1}\right)=1$ if $\tilde{\mathbf{g}}$ is orthogonal to the MAI subspace $\mathcal{S}_{I}$ and $\eta\left(\mathbf{g}_{1}\right)=0$ if $\tilde{\mathbf{g}} \in \mathcal{S}_{I}$. In general, as long as $N_{\text {tot }} \geq$ $N_{i}+1$ and $\tilde{\mathbf{g}}_{\perp} \neq \mathbf{0}$, the system is near-far resistant.

As evident from (40), near-far resistance is also a measure of the transmitted power required in a single-user receiver, relative to that in a multiuser receiver, to achieve identical performance. The value of $0 \leq \eta\left(\mathbf{g}_{1}\right) \leq 1$ reflects the relative loss of efficiency in a multiuser receiver in terms of the transmitted power at high SNRs. We now investigate the relative powers required in single-user and multiuser systems over time scales much longer than $T_{\mathrm{coh}, d}$. For this comparison, the $P_{e}$ of the single-user receiver is given by averaging $Q\left(\sqrt{2 \| \mathrm{g}_{1}||^{2} / \sigma^{2}}\right)$ over the distribution of $\mathbf{g}_{1}$. Following the discussion in Section IV-B, the $P_{e}$ is governed by the eigenvalues of $\mathbf{R}_{g_{1}}$, say

\footnotetext{
${ }^{14}$ Parallel to a similar result in [18] for AWGN channels.
} 
$\left\{\mu_{s u, l}: l=1,2, \ldots, D_{p}\right\}$, and is given by (38) with $\mu_{l}=$ $\mu_{s u, l} / \sigma^{2}$. Similarly, recall that the $P_{e}$ of the multiuser receiver is governed by the eigenvalues of $\mathbf{R}_{\epsilon \epsilon}^{-1} \mathbf{R}_{g_{1}}$. From (36) we note that as $\sigma^{2} \rightarrow 0, \sigma^{2} \mathbf{R}_{\epsilon \epsilon}^{-1} \approx \mathbf{P}_{\perp, p}=\mathbf{V}_{\perp, p} \mathbf{V}_{\perp, p}^{H}$. Thus, in the limit of high SNR, the $P_{e}$ of the multiuser receiver is governed by the eigenvalues of $\mathbf{P}_{\perp, p} \mathbf{R}_{g_{1}}$, say $\left\{\mu_{m u, l}\right\}$, and is given by (38) with $\mu_{l}=\mu_{m u, l} / \sigma^{2}$. Furthermore, for high SNR $(\mu \gg 1)$, the expression for $P_{e}$ in (38) can be simplified as [1] $P_{e} \approx$ $\left(\begin{array}{c}2 D_{p}-1 \\ D_{p}\end{array}\right) \prod_{l=1}^{D_{p}}\left(1 / 4 \mu_{l}\right)$. Let $r$ denote the transmitted power of the single-user receiver relative to the multiuser receiver. It follows from the above approximate expression for $P_{e}$ that

$$
\frac{P_{e}(\text { multiuser })}{P_{e}(\text { single user })} \approx r^{D_{p}} \prod_{l=1}^{D_{p}} \frac{\mu_{s u, l}}{\mu_{m u, l}} .
$$

Thus, for identical $P_{e}$ in the two systems as $\sigma^{2} \rightarrow 0$, the relative power of the single-user (MRC) receiver should be $r=$ $\left[\prod_{l=1}^{D_{p}}\left(\mu_{m u, l} / \mu_{s u, l}\right)\right]^{1 / D_{p}}$. We note that typically $r \leq 1$ since the eigenvalues of $\mathbf{P}_{\perp, p} \mathbf{R}_{g_{1}}$ (multiuser) are typically smaller than the eigenvalues of $\mathbf{R}_{g_{1}}$ (single user) since $\mathbf{P}_{\perp, p}$ maps to a lower-dimensional space. Moreover, as $D_{p}$ increases $r \rightarrow 1$. Thus, for a sufficiently large dimension of the primary subspace, and under sufficiently high SNR, we expect the $P_{e}$ of the multiuser receiver to be very close to that of a single-user MRC receiver operating in the absence of MAI. This trend is exhibited in Fig. 6 (discussed in Section V).

\section{Choice of Secondary Coordinates}

There are two key issues in this context: the number $D_{s}$ of secondary coordinates and the choice of the $D_{s}$ coordinates from amongst all the inactive coordinates possible. The choice of $D_{s}$ is primarily guided by the dimensional analysis in Section IV-A on performance analysis. The main conclusion is that at high SNR, we need $D_{s} \approx N_{i}+1-D_{p}$ to adequately suppress MAI with $N_{i}$ degrees of freedom. As the noise level increases, increasing $D_{s}$ would in general improve performance, however, beyond a certain point, we expect to see diminishing returns, as we will see in the next section. For any given $D_{s}$ coordinates, the MMSE receiver is given by $\mathbf{w}_{o}=\mathbf{R}_{z z}^{-1} \tilde{\mathbf{g}}$ which we have seen in several forms.

Given $D_{s}$, the choice of the "best" $D_{s}$ inactive coordinates is in general a difficult one: it depends on the correlation structure of primary and secondary coordinates and it involves $\left(\begin{array}{c}N_{o}-D_{p} \\ D_{s}\end{array}\right)$ possibilities. One brute force approach is to choose the set of $D_{s}$ coordinates that minimizes the trace of the resulting error covariance matrix $\mathbf{R}_{\mathrm{ee}}$ in estimating $\mathbf{z}_{p}$ from $\mathbf{z}_{s}$. While a thorough discussion of this issue is beyond the scope of this paper, we note that the difference in performance between different choices may not be very significant as illustrated in Fig. 8 (discussed in the next section).

\section{ILLUSTRATIVE EXAMPLES}

We now illustrate various features of the framework with numerical examples. We consider a system that employs Gold codes for all users with a spreading gain of $N=63$. The system supports $K=4$ users in a relatively slow fading environment. A wide-sense stationary uncorrelated scattering (WSSUS) channel is simulated via the model (3) using 16 uniformly spaced paths per chip duration with independent, equal-power fading coefficients $\left(\mathrm{E}\left[\mathbf{h}_{k} \mathbf{h}_{k}^{H}\right]=P_{k} \mathbf{I}\right.$, where $P_{k}$ is the power of the $k$ th user). All users experience a multipath spread of $T_{m}=2 T_{c}$ and negligible Doppler effects within a symbol duration $\left(T B_{d} \approx 0 ; M=0\right)$. An active basis corresponding to fourfold oversampling $\left[B=4 / T_{c}\right.$ in (4)] is employed for the front-end processing at the receiver [see (5)]. This corresponds to $D_{p}=9$ active coordinates for each user. The background noise is such that the SNR of the desired user $\left(\mathrm{E}\left[2\left\|\mathrm{~g}_{1}\right\|^{2} / \sigma^{2}\right]\right)$ is $20 \mathrm{~dB}$ unless otherwise noted. All results correspond to BPSK signaling and channel coefficients of the desired user are assumed known at the receiver.

Fig. 3 illustrates the improvement in SINR as a function of $D_{s}$. We consider the case where $T_{\mathrm{obs}} \gg T_{\mathrm{coh}, i}$ but $T_{\mathrm{obs}}<$ $T_{\text {coh, } d}$. Thus, $N_{i}=(K-1) D_{p}$ whereas the desired user exhibits a fixed direction over $T_{\text {obs }}$. This artificial difference between the coherence times of the desired and interfering users is used for illustrative purposes to simulate a system with a large number of interference degrees of freedom. The SINR is plotted as a function of the relative power $P$ of each interfering user. ${ }^{15}$ Fig. 3(a) corresponds to $K-1=1$ resulting in $N_{i}=D_{p}=9$ MAI degrees of freedom. Fig. 3(b) corresponds to $K-1=3$ resulting in $N_{i}=27 .{ }^{16}$ Note the performance of the MMSE receiver with $D_{s}=0$ is significantly better than that of the MRC receiver. Furthermore, the performance of the MMSE receiver increases significantly as $D_{s}$ is increased until $N_{\text {tot }}=D_{p}+D_{s}$ approaches $N_{i}+1$, beyond which the performance saturates.

Fig. 4 illustrates the variation in SINR as a function of the SNR of desired user for different $D_{s}$. There are three interfering users with the same power as the desired user. Fig. 4(a) depicts the case where $T_{\mathrm{obs}}<T_{\mathrm{coh}, i}$ resulting in $N_{i}=3$, whereas Fig. 4(b) corresponds to the $T_{\mathrm{obs}} \gg T_{\mathrm{coh}, i}$ case resulting in $N_{i}=27$. The figure clearly shows two regimes depending on $D_{s}$ : MAI-limited regime when $D_{s}+D_{p}<N_{i}+1$ and noise-limited regime when $D_{s}+D_{p} \geq N_{i}+1$. While the SINR saturates in the MAI-limited regime, it increases linearly with SNR in the noise-limited regime. For fixed value of $N_{i}$, the system moves from an MAI-limited regime to a noise-limited one as $D_{s}$ is increased. For example, in Fig. 4(b), $N_{i}=27$ and the system is MAI-limited for $D_{s}<28-9=19$ as evident from the curves for $D_{s}=0,4$. For $D_{s} \geq 19$, it is noise limited as evident from the curves for $D_{s}=20,30$.

Fig. 5 shows the analytically computed $P_{e}$ [via (38)] as a function of the relative power of each interfering user for different values of $D_{s}$. In this case, there are $K-1=3$ interfering users with $T_{\mathrm{obs}} \gg T_{\mathrm{coh}, i}$ resulting in $N_{i}=27$. The improvement in performance with increasing $D_{s}$ is evident. Fig. 6 explores the validity of the $P_{e}$ expression in (38) for a variety of scenarios. Analytical computation of $P_{e}$ in (38) is compared with Monte-Carlo averaging of the conditional $P_{e}$ expression in (37) over 2000 channel realizations of all four users. Fig. 6(a) and (b) shows the comparison for $D_{s}=1$ and $D_{s}=20$ in the case when all users have equal power. Fig. 6(c)-(f) shows the

\footnotetext{
${ }^{15}$ We assume the same relative power $P$ for each interfering user: $\mathrm{E}\left[\mathbf{h}_{1} \mathbf{h}_{1}\right]=$ $\mathbf{I}$ and $\mathrm{E}\left[\mathbf{h}_{k}, \mathbf{h}_{k}^{H}\right]=P \mathbf{I}$.

${ }^{16}$ The two scenarios may be interpreted as representing 9 versus 27 interfering users when $T_{\text {obs }}<T_{\text {coll, } i \text {. }}$.
} 


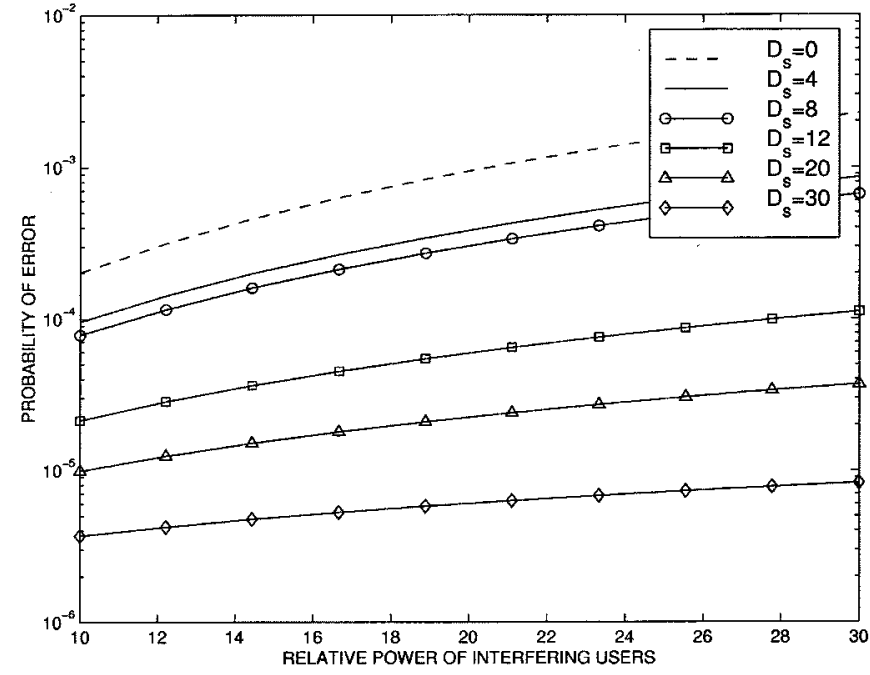

Fig. 5. Analytically computed $P_{e}$ [using (38)] as a function of the relative power of three interfering users $\left(N_{i}=27\right)$ for different values of $D_{s}$.

comparison between $D_{s}=1$ and $D_{s}=40$ for higher interference powers. Fig. 6(c) and (d) shows the comparison for the case when the interfering users are three times stronger, and Fig. 6(e) and (f) depicts the case when the interfering users are ten times stronger. Consistent with the conclusions drawn in [20], the approximation in (38) is fairly good and is more accurate when effective MAI in the output of the receiver is relatively small (which is true for large $D_{s}$ ). We note that the analytical approximation in (38) seems to lower bound the actual receiver performance.

Fig. 7 plots the the near-far resistance [via (41)] versus $D_{s}$ for three different realizations of $\mathbf{h}_{\boldsymbol{k}}$ 's of all users to illustrate variation in performance with channel coefficients. There are three interfering users with $T_{\mathrm{obs}}<T_{\mathrm{coh}, i}\left(N_{i}=3\right)$ and each one is five times stronger than the desired user. Note that the value of $\eta$ tends to converge with increasing $D_{s}$ and the improvement in near-far resistance saturates as $D_{s}$ is increased.

Finally, Fig. 8 explores the effect of the choice of secondary coordinates on performance. There are three strong interfering users with $T_{\mathrm{obs}} \gg T_{\mathrm{coh}, i}\left(N_{i}=27\right)$ and $P_{e}$ is analytically computed for four different choices of secondary coordinates as a function of the relative power of each interfering user. Fig. 8(a) corresponds to $D_{s}=12$ and Fig. 8 (b) corresponds to $D_{s}=30$. The best attainable performance, that of the full-dimensional $\left(D_{s}=N-D_{p}\right)$ receiver, is also plotted for comparison. Notice that the difference in performance for the four different choices is not too significant, especially for the larger value of $D_{s}$.

\section{IMPLEMENTATION ISSUES}

The proposed MMSE receiver structure requires knowledge of second-order statistics $\left(\mathbf{R}_{z z}\right)$ of the canonical coordinates and the channel coefficients of the desired user $\mathbf{h}_{1}$. In practice, both of these quantities have to be estimated from data. In this section, we briefly discuss such implementational issues. We first discuss adaptive formulations of the receiver which assume the knowledge of $\mathbf{h}_{1}$ and infer the optimum solution directly from data by implicitly estimating $\mathbf{R}_{z z}$. We then discuss the estimation of $\mathbf{h}_{1}$ directly from data using one of the adaptive receiver structures.

\section{A. Adaptive Implementations}

To derive an adaptive formulation of the proposed receiver, we first cast it in the framework of linearly constrained minimum variance (LCMV) filtering [21]. In this formulation, the optimum filter $\mathbf{w}_{m v, o}$ minimizes the average output power subject to constraining the signal component to remain constant

$$
\mathbf{w}_{m v}=\arg \min _{\mathbf{w}} \mathrm{E}\left[\left|\mathbf{w}^{H} \mathbf{z}\right|^{2}\right] \quad \text { subject to } \mathbf{w}_{p}^{H} \mathbf{g}_{1}=\alpha .
$$

Using the method of Lagrange multipliers [21], the solution to (43) is given by

$$
\begin{aligned}
\mathbf{w}_{m v}\left(\mathbf{g}_{1}\right) & =\frac{\alpha}{\tilde{\mathbf{g}}_{1}^{H} \mathbf{R}_{z z}^{-1} \tilde{\mathbf{g}}_{1}} \mathbf{R}_{z z}^{-1} \tilde{\mathbf{g}}_{1}=\frac{\alpha}{\mathbf{g}_{1}^{H} \mathbf{R}_{e e}^{-1} \mathbf{g}_{1}} \mathbf{R}_{e e}^{-1} \mathbf{g}_{1} \\
& =\frac{\alpha}{\mathbf{g}_{1}^{H} \mathbf{R}_{\epsilon \epsilon}^{-1} \mathbf{g}_{1}}\left[\begin{array}{c}
\mathbf{R}_{\epsilon \epsilon}^{-1} \mathbf{g}_{1} \\
-\mathbf{C R}_{\epsilon \epsilon}^{-1} \mathbf{g}_{1}
\end{array}\right] \\
m v\left(\mathbf{g}_{1}\right) & =\frac{\alpha^{2}}{\mathbf{g}_{1}^{H} \mathbf{R}_{e e}^{-1} \mathbf{g}_{1}}=\frac{\alpha^{2}\left(1+\mathbf{g}_{1}^{H} \mathbf{R}_{\epsilon \epsilon}^{-1} \mathbf{g}_{1}\right)}{\mathbf{g}_{1}^{H} \mathbf{R}_{\epsilon \epsilon}^{-1} \mathbf{g}_{1}}
\end{aligned}
$$

Note that the solutions in (44) and (28) are identical up to a scalar.

To implement $\mathbf{w}_{m v}$ adaptively, we formulate it as a generalized sidelobe canceler (GSC) [21] to convert the constrained problem in (43) into an unconstrained one. Fig. 9 shows two adaptive implementations based on the GSC approach. Fig. 9(a) shows a parallel scheme, along the lines of Fig. 1(a) in which $\mathbf{w}_{p}$ and $\mathbf{w}_{s}$ are adapted simultaneously ( $D_{p}+D_{s}$ adaptive taps). Whereas the secondary filter $\mathbf{w}_{s}$ is adapted unconstrained, the primary filter is decomposed as $\mathbf{w}_{p}=a \mathbf{g}_{1}+\mathbf{w}_{\perp}$ to satisfy the constraint in (43). In particular, $\mathbf{w}_{\perp}=\mathbf{G}^{\perp} \mathbf{w}_{u c}$ operates on the orthogonal complement of $\mathbf{g}_{1}$, which is spanned by the columns of the $D_{p} \times\left(D_{p}-1\right)$ matrix $\mathbf{G}^{\perp}$. This is illustrated in Fig. 9(a) in which $\mathbf{w}_{u c}\left(D_{p}-1\right.$ taps) and $\mathbf{w}_{s}\left(D_{s}\right.$ taps) represent the unconstrained degrees of freedom that can be adaptively updated using any standard adaptative algorithm such as least mean squares or recursive least squares [21].

Fig. 9(b) shows a sequential implementation along the lines of Fig. 1(b) in which the MAI component in $\mathbf{z}_{p}$ is first estimated from $\mathbf{z}_{s}$ via a matrix filter $\mathbf{C}$ (or a bank of filters). The primary filter $\mathbf{w}_{p}$ then acts on the error signal $\mathbf{e}$ to further suppress any residual interference. Note that $\mathbf{C}$ can be adapted unconstrained since the secondary coordinates are signal free. The primary filter $\mathbf{w}_{p}$ is decomposed as in the parallel scheme to satisfy the constraint. In this case, the $\mathbf{w}_{u c}$ and $\mathbf{C}$ represent the $\left(D_{p}-\right.$ 1) $+\left(D_{p} \times D_{s}\right)$ unconstrained degrees of freedom that can be adaptively updated. A thorough analysis of these adaptive implementations is beyond the scope of this paper. However, we note that the serial scheme in Fig. 9(b) is particulary suited for blind estimation of $\mathbf{g}_{1}$, as discussed in the next section. Under certain conditions, the dominant eigenvector of $\mathbf{R}_{e e}$ yields an accurate estimate of $\mathbf{g}_{1}$.

\section{B. Channel Estimation}

If $\mathbf{h}_{1}$ is not known a priori, we can extend the LCMV formulation analogous to the use of Capon's method in [8] to estimate the channel coefficients. We outline an approach for estimating $\mathbf{g}_{1}=\mathbf{Q}_{11} \mathbf{h}_{1}$ from which $\mathbf{h}_{1}$ can be obtained since $\mathbf{Q}_{11}^{-1}$ exists. It is based on the serial adaptive receiver in Fig. 9(b). 


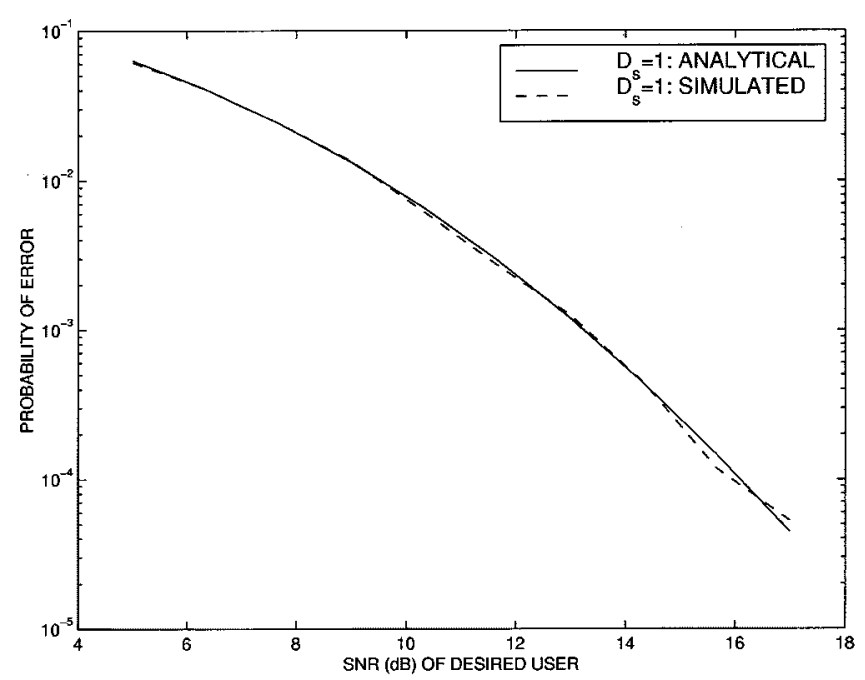

(a)

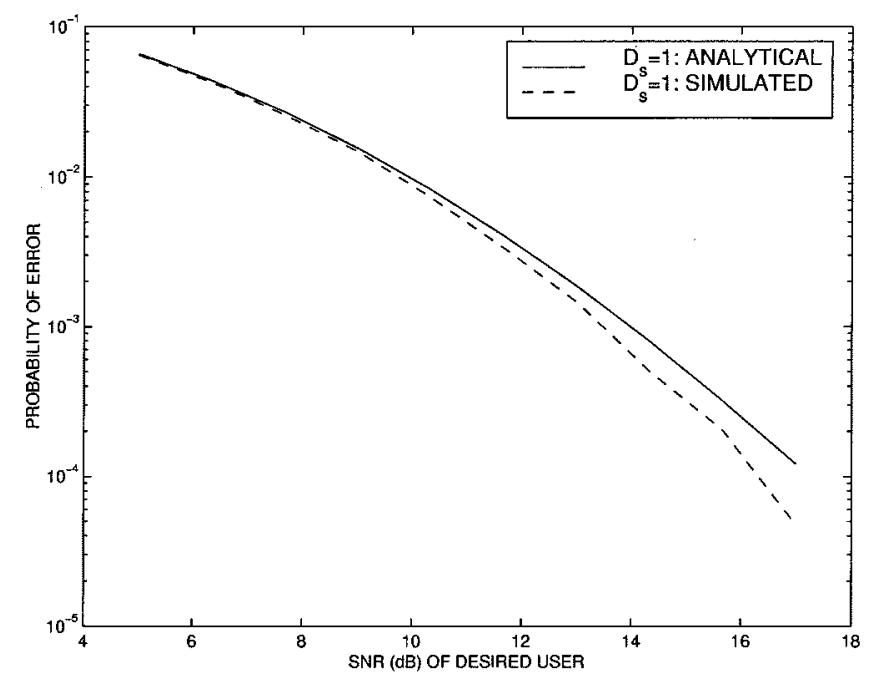

(c)

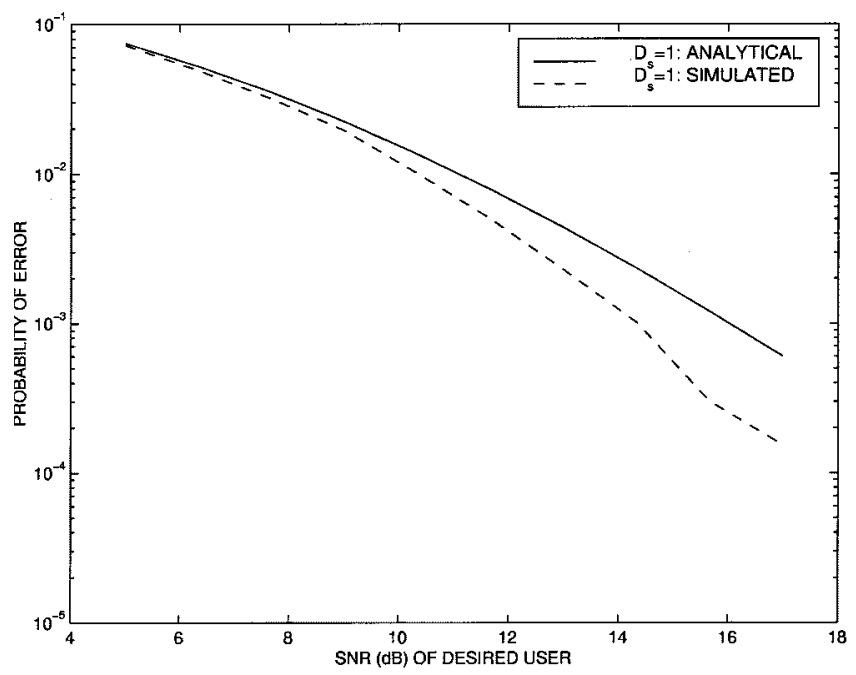

(e)

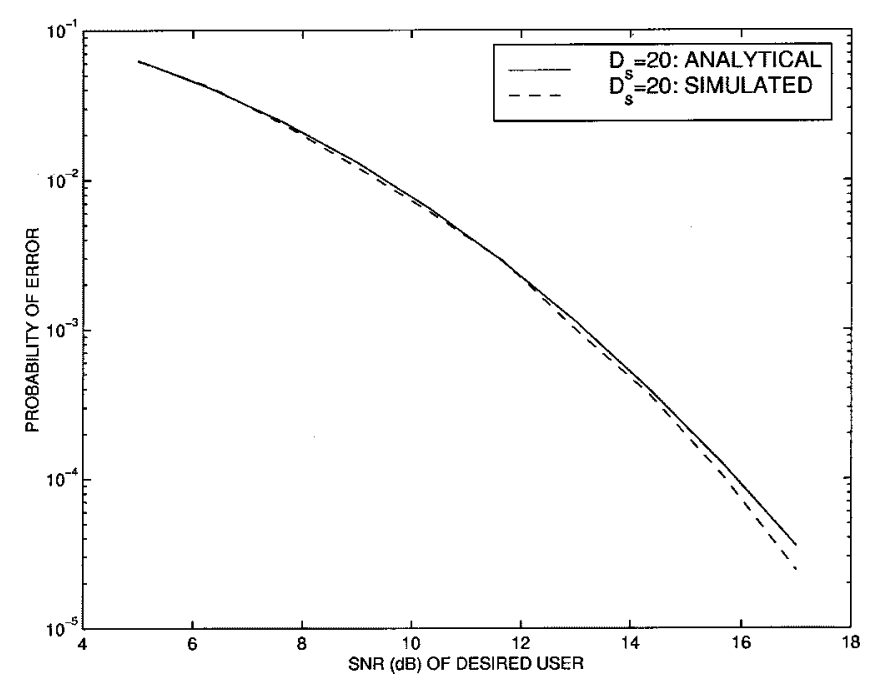

(b)

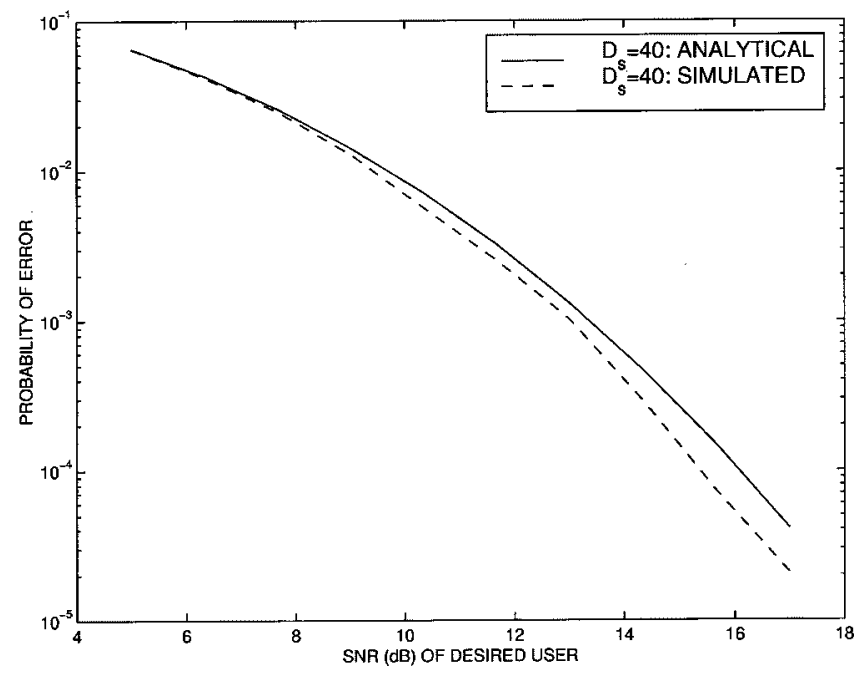

(d)

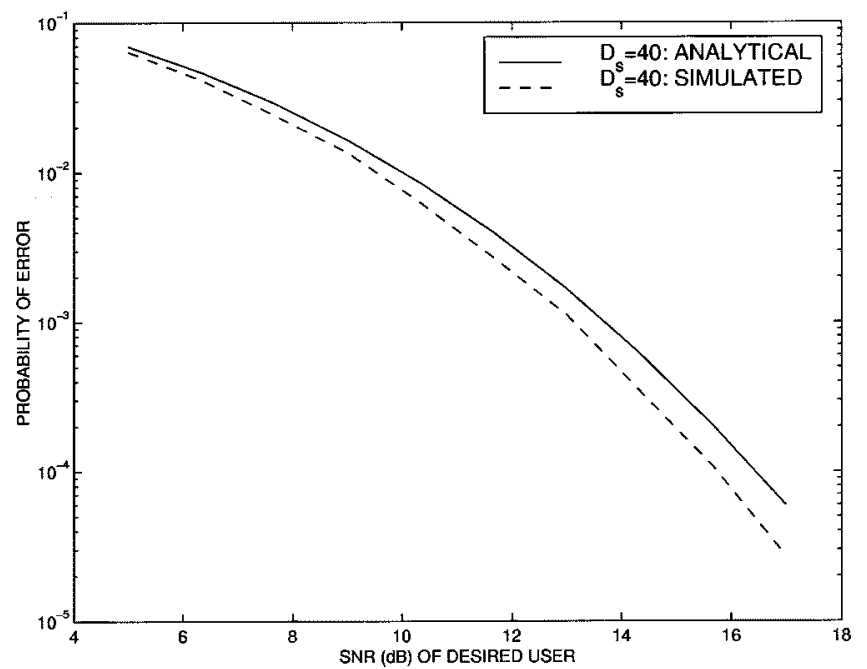

(f)

Fig. 6. Comparison of analytically computed $P_{e}$ [via (38)] versus Monte-Carlo averaging of (37) over all $\mathbf{h}_{k}$ 's for different values of $D_{s}$. Three interfering users. (a) and (b): all users with equal power. (c) and (d): interfering users are three times stronger than desired user. (e) and (f): interfering users are ten times stronger. We note for large $D_{s}$ the receiver performance is close to that of a single-user (MRC) receiver with three-level diversity [1] operating in the absence of MAI.

We start by noting that the constraint in the LCMV approach is based on $\mathbf{g}_{1}$. In the extended formulation, we replace $\mathbf{g}_{1}$ with an arbitrary constraint vector $f_{1}$. This yields the corresponding LCMV solution in (44) and (45) in terms of $\mathbf{f}_{1}$. Then, the op- 


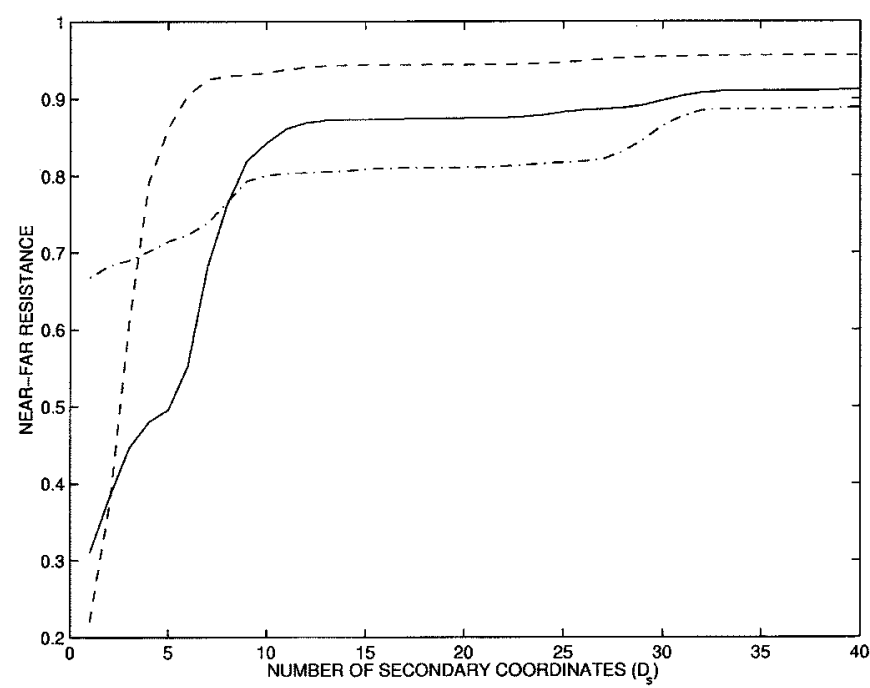

Fig. 7. Near-far resistance, computed using (41) for three different realizations of $\mathbf{h}_{k}$ 's, as a function of $D_{\mathcal{s}}$. There are three interfering users that are five times stronger than the desired user.

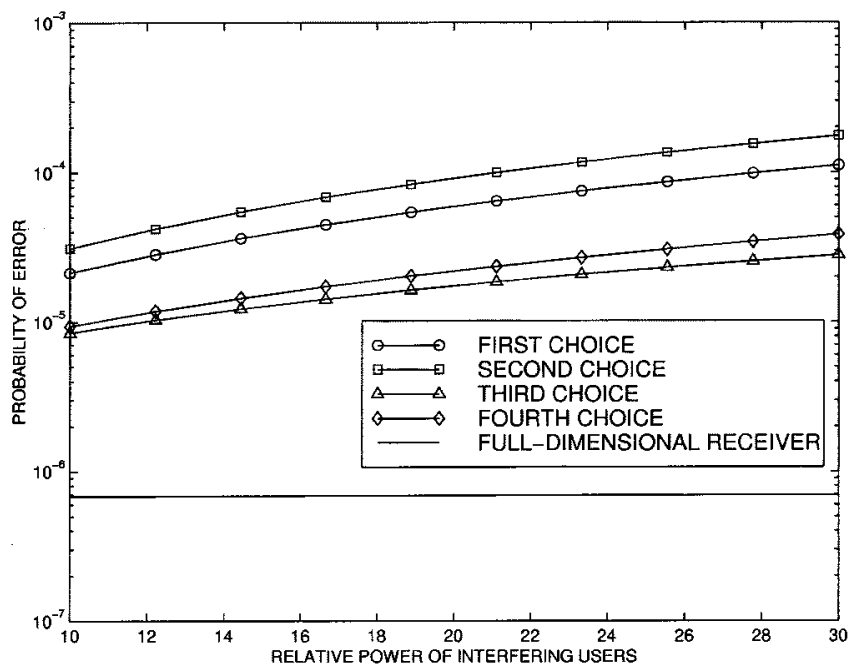

(a)

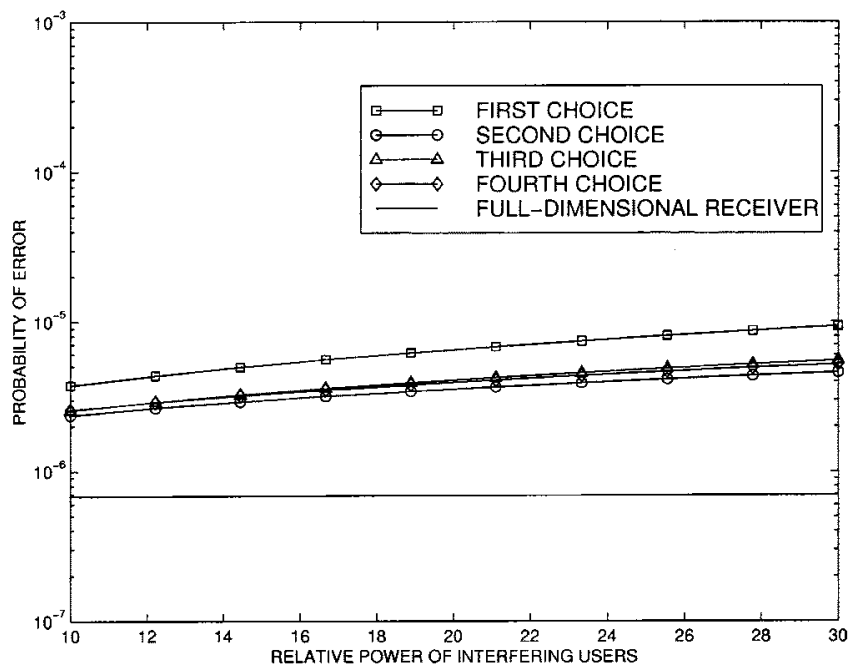

(b)

Fig. 8. Analytically computed $P_{e}$ for different choices of secondary coordinates as a function of the relative power of three interfering users. (a) $D_{s}=12$. (b) $D_{s}=30$. Notice that the relatively small difference in performance between different choices, especially for larger $D_{s}$.

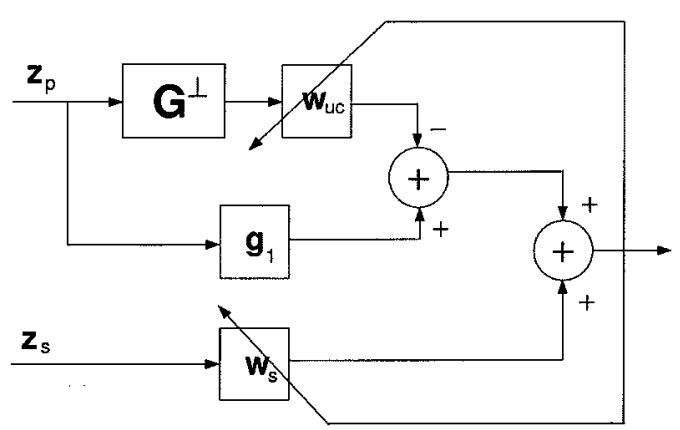

(a)

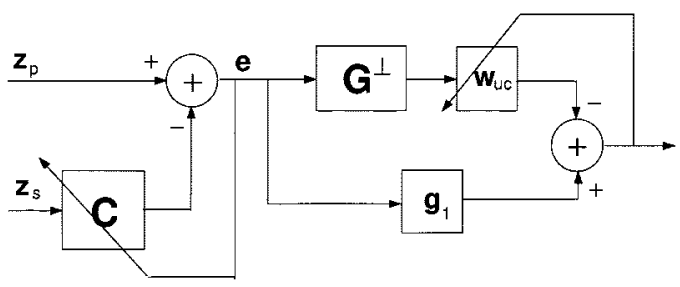

(b)

Fig. 9. Structures for adaptive implementation: (a) parallel structure and (b) serial structure. The serial structure is particularly suited to blind channel estimation from the eigenstructure of $\mathbf{e}$.

timum (unit-norm) $\mathrm{f}_{1}$ is defined as one which maximizes the parameterized minimum output variance

$$
\begin{aligned}
\mathbf{f}_{1, o} & =\arg \max _{\left\|\mathbf{f}_{1}\right\|=1} \min _{\mathbf{w}: \mathbf{w}_{p}^{H} \mathbf{f}_{1}=\alpha} \mathbf{w}^{H} \mathbf{R}_{z z} \mathbf{w} \\
& =\arg \min _{\left\|\mathbf{f}_{1}\right\|=1} \mathbf{f}_{1}^{H} \mathbf{R}_{\mathrm{ee}}^{-1} \mathbf{f}_{1}
\end{aligned}
$$

where we have used (45) in the last equality. The solution to (46) is given by the eigenvector corresponding to the smallest eigenvalue of $\mathbf{R}_{\mathrm{ee}}^{-1}$ (or largest eigenvalue of $\mathbf{R}_{\mathrm{ee}}$ ). ${ }^{17}$ This solution yields an estimate of $\mathbf{h}_{1}$ as $\hat{\mathbf{h}}_{1}=\mathbf{Q}_{11}^{-1} \mathbf{f}_{1, o}$. Furthermore, the corresponding MMSE/LCMV solution is given by $\mathbf{w}_{m v}\left(\mathbf{f}_{1, o}\right)$ in (44).

Note that $\mathbf{e}=\mathbf{g}_{1}+\Delta \mathbf{i}_{p}+\Delta \mathbf{n}_{p}$, where $\Delta \mathbf{i}_{p}$ and $\Delta \mathbf{n}_{p}$ represent the residual MAI and noise after the first stage of MAI suppression effected by $\mathbf{C}$, as illustrated in Fig. 9(b). Intuitively, for sufficiently high SNR and for sufficiently many secondary coordinates, we expect the dominant eigenvector of $\mathbf{R}_{\mathrm{ee}}$ to be strongly influenced by $\mathbf{g}_{1}$. It is shown in the Appendix that a necessary condition for

$$
\lim _{\sigma^{2} \rightarrow 0} \mathbf{f}_{1, o}=\frac{e^{j \phi} \mathbf{g}_{1}}{\left\|\mathbf{g}_{1}\right\|}, \quad \phi \in \mathbb{R} \text { (phase ambiguity) }
$$

is that $D_{s} \geq N_{i}$ (secondary coordinates alone are sufficient to completely suppress the MAI). We note that in our simulations we have found this condition to be sufficient as well. Thus, the dominant eigenvector of $\mathbf{R}_{e e}$ yields a reliable channel estimate up to a scale factor. A representative plot of $\log \left(\| \mathrm{f}_{1, o}-\right.$ $e^{j \phi} \mathbf{g}_{1} /\left\|\mathbf{g}_{1}\right\| \|^{2}$ ), where $\mathbf{f}_{1, \circ}$ is computed using (46), is shown in Fig. 10 as a function of SNR for different values of $D_{s}$. There are three interfering users with $T_{\mathrm{obs}}<T_{\mathrm{coh}, i}$. It is evident that the estimation error decreases monotonically with SNR for $D_{s} \geq N_{i}=3$.

${ }^{17}$ Assuming that the largest eigenvalue of $\mathbf{R}_{\mathrm{cc}}$ corresponds to a one-diminsional invariant subspace. Otherwise, any vector in the invariant subspace will suffice. 


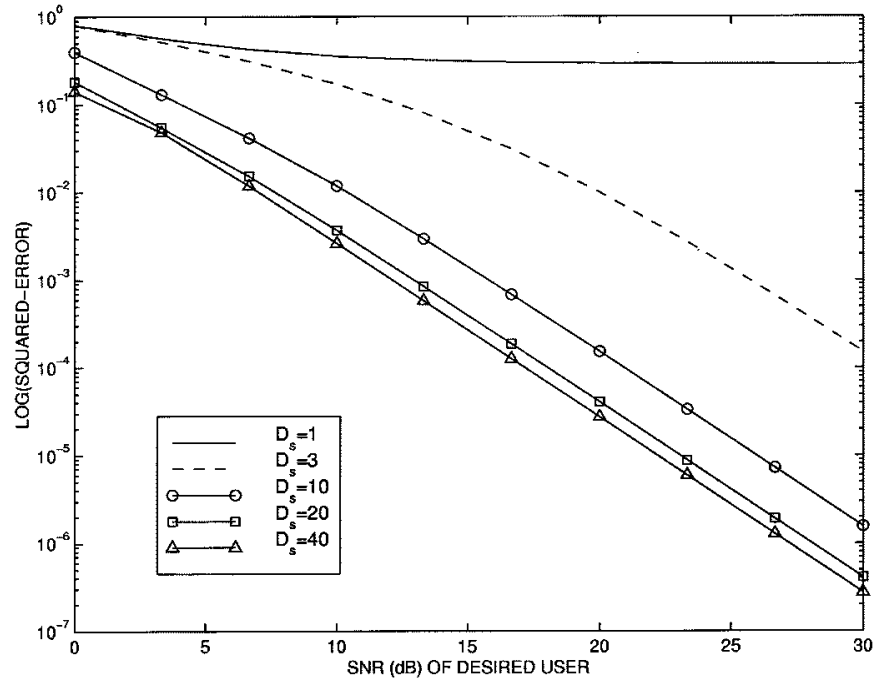

Fig. 10. A representative plot of the channel estimation error as a function of SNR of desired user for different value of $D_{s}$. Three interfering users that are ten times stronger than desired user. Notice the monotonic decrease in error for $D_{s} \geq N_{i}=3$.

Recall that the MMSE and LCMV solutions are identical up to a scalar and note from (33) that in the limit of high SNR, the optimum primary solution $\mathbf{w}_{p, o}=\mathbf{R}_{e e}^{-1} \mathbf{g}_{1}=c \mathbf{g}_{1, \perp}$. However, in the limit $\mathbf{g}_{1}$ is an eigenvector of $\mathbf{R}_{\mathrm{ee}}$. Thus, we expect that in the limit of high SNR the optimum primary solution is identical to $\mathrm{g}_{1}$ as long as $D_{s} \geq N_{i}$.

\section{CONCLUSION}

The generic linear multiuser receiver in Fig. 1(a) typifies our integrated design framework in terms of canonical multipathDoppler coordinates. The canonical coordinates fully incorporate time-varying channel dispersion effects via the robust and parsimonious signal representation (3) and provide a natural subspace approach to exploit dispersion effects for MAI suppression. The primary coordinates facilitate maximal exploitation of channel diversity and limited MAI suppression. Progressively enhanced MAI suppression can be attained by augmenting the receiver with secondary coordinates. The framework also lends itself easily to blind/adaptive implementations that require knowledge of only the desired user's code.

The natural signal space partitioning in terms of primary and secondary coordinates provides a direct handle on controlling receiver complexity. This is a particularly attractive feature in adaptive implementations in which the desired input statistics have to be estimated directly from data. For example, as reported in [7], existing chip-rate sampled adaptive receiver designs operating in the full-dimensional signal space suffer from poor performance due to unreliable estimation of data covariance matrix in practical time-varying scenarios. Our framework for designing a range of progressively complex (powerful) receivers by incorporating secondary coordinates serves as a useful approach for striking a judicious practical tradeoff between complexity and performance. Furthermore, while our examples focused on slow fading scenarios, the notion of Doppler diversity with appropriate signaling [13], [23] may also be leveraged in fast-fading scenarios to further enhance system performance.
Finally, we note that notion of canonical coordinates can be extended to space-time scenarios as well [24], [25].

\section{APPENDIX}

Recall that $\mathbf{R}_{\mathrm{ee}}=\mathbf{g}_{1} \mathbf{g}_{1}^{H}+\mathbf{R}_{\epsilon \epsilon}$. It follows from the matrix inversion lemma [21] that:

$$
\mathbf{R}_{e e}^{-1}=\mathbf{R}_{\epsilon \epsilon}^{-1}-\frac{\mathbf{R}_{\epsilon \epsilon}^{-1} \mathbf{g}_{1} \mathbf{g}_{1}^{H} \mathbf{R}_{\epsilon \epsilon}^{-1}}{1+\mathbf{g}_{1}^{H} \mathbf{R}_{\epsilon \epsilon}^{-1} \mathbf{g}_{1}}
$$

To characterize the eigenvector corresponding to the minimum eigenvalue, we study the Rayleigh quotient $\left(\mathbf{x}^{H} \mathbf{R}_{e e}^{-1} \mathbf{x}\right) /\|\mathbf{x}\|^{2}$. From (48), it follows that

$$
\begin{aligned}
\mathbf{x}^{H} \mathbf{R}_{e e}^{-1} \mathbf{x}= & \frac{\sigma^{2} \mathbf{x}^{H} \mathbf{R}_{\epsilon \epsilon}^{-1} \mathbf{x}}{\sigma^{2}+\sigma^{2} \mathbf{g}_{1}^{H} \mathbf{R}_{\epsilon \epsilon}^{-1} \mathbf{g}_{1}} \\
& +\frac{\sigma^{2}\left(\mathbf{g}_{1}^{H} \mathbf{R}_{\epsilon \epsilon}^{-1} \mathbf{g}_{1}\right)\left(\mathbf{x}^{H} \mathbf{R}_{\epsilon \epsilon}^{-1} \mathbf{x}\right)}{\sigma^{2}+\sigma^{2} \mathbf{g}_{1}^{H} \mathbf{R}_{\epsilon \epsilon}^{-1} \mathbf{g}_{1}} \\
& -\frac{\sigma^{2}\left|\mathbf{x}^{H} \mathbf{R}_{\epsilon \epsilon}^{-1} \mathbf{g}_{1}\right|^{2}}{\sigma^{2}+\sigma^{2} \mathbf{g}_{1}^{H} \mathbf{R}_{\epsilon \epsilon}^{-1} \mathbf{g}_{1}} .
\end{aligned}
$$

Recall from (27) that

$$
\mathbf{R}_{\epsilon \epsilon}^{-1}=\mathbf{V}_{I, p} \tilde{\Lambda}^{-1} \mathbf{V}_{I, p}^{H}+\frac{1}{\sigma^{2}} \mathbf{V}_{\perp, p} \mathbf{V}_{\perp, p}^{H}
$$

from which we conclude that as $\sigma^{2} \rightarrow 0, \mathbf{R}_{\epsilon \epsilon}^{-1} \approx\left(1 / \sigma^{2}\right) \mathbf{P}_{\perp, p}$ $=\left(1 / \sigma^{2}\right) \mathbf{V}_{\perp, p} \mathbf{V}_{\perp, p}^{H}$. Combining this fact with (49), we conclude that for $\sigma^{2} \stackrel{\perp}{\rightarrow} 0$

$$
\begin{aligned}
\mathbf{x}^{H} \mathbf{R}_{e e}^{-1} \mathbf{x} \approx & \frac{\mathbf{x}^{H} \mathbf{P}_{\perp, p} \mathbf{x}}{\sigma^{2}+\mathbf{g}_{1}^{H} \mathbf{P}_{\perp, p} \mathbf{g}_{1}} \\
& +\frac{1}{\sigma^{2}} \frac{\left(\mathbf{g}_{1}^{H} \mathbf{P}_{\perp, p} \mathbf{g}_{1}\right)\left(\mathbf{x}^{H} \mathbf{P}_{\perp, p} \mathbf{x}\right)}{\sigma^{2}+\mathbf{g}_{1}^{H} \mathbf{P}_{\perp, p} \mathbf{g}_{1}} \\
& -\frac{1}{\sigma^{2}} \frac{\left|\mathbf{x}^{H} \mathbf{P}_{\perp, p} \mathbf{g}_{1}\right|^{2}}{\sigma^{2}+\mathbf{g}_{1}^{H} \mathbf{P}_{\perp, p} \mathbf{g}_{1}} .
\end{aligned}
$$

From (51), we conclude that in order to keep $\mathbf{x}^{H} \mathbf{R}_{e e}^{-1} \mathbf{x}$ from blowing up (as $\sigma^{2} \rightarrow 0$ ), we need to have the second and third terms on the right-hand side equal to zero. Clearly, the choice $\mathbf{x}=\alpha \mathbf{g}_{1}$ accomplishes this and it follows that

$$
\lim _{\sigma^{2} \rightarrow 0} \frac{\alpha \mathbf{g}_{1}^{H} \mathbf{R}_{e e}^{-1} \alpha \mathbf{g}_{1}}{\left\|\alpha \mathbf{g}_{1}\right\|^{2}}=\frac{1}{\left\|\mathbf{g}_{1}\right\|^{2}} .
$$

However, $\mathbf{X}=\mathbf{g}_{1}$ may not be the unique choice unless $\mathbf{P}_{\perp, p}$ has rank $D_{p}$ (full rank). Since $\mathbf{V}_{\perp, p}$ is $D_{p} \times\left(N_{\text {tot }}-N_{i}\right)$, a necessary condition for $\mathbf{P}_{\perp, p}$ to be full rank is that $N_{\text {tot }}-N_{i}=D_{p}+D_{s}-$ $N_{i} \geq D_{p}$, or equivalently, $D_{s} \geq N_{i}$. Thus, we conclude that a necessary condition for the least (most) dominant eigenvector of $\mathbf{R}_{e e}^{-1}\left(\mathbf{R}_{e e}\right)$ to converge to $e^{j \phi} \mathbf{g}_{1} /\left\|\mathbf{g}_{1}\right\|$ in the limit of high SNR (with $1 /\left\|\mathbf{g}_{1}\right\|^{2}$ as the corresponding eigenvalue) ${ }^{18}$ is that $D_{s} \geq N_{i}$. This proves (47).

\section{ACKNOWLEDGMENT}

The authors would like to acknowledge Prof. L. Scharf, Prof. B. Van Veen, and E. Onggosanusi for useful discussions during the course of this work.

\footnotetext{
${ }^{18}$ The remaining $D_{p}-1$ eigenvalues of $\mathbf{R}_{\epsilon e}^{-1}\left(\mathbf{R}_{\mathrm{cc}}\right) \rightarrow+\infty(0)$.
} 


\section{REFERENCES}

[1] J. G. Proakis, Digitial Communications, 3rd ed. New York: McGrawHill, 1995.

[2] D. Slepian, "On bandwidth," Proc. IEEE, vol. 64, pp. 292-300, Mar. 1976.

[3] S. Verdu, Multiuser Detection. Cambridge, U.K.: Cambridge Univ. Press, 1998

[4] H. V. Poor and G. W. Wornell, Eds., Wireless Communications: Signal Processing Perspectives. Englewood Cliffs, NJ: Prentice-Hall, 1998.

[5] U. Madhow, "Blind adaptive interference suppression for direct-sequence CDMA," Proc. IEEE, vol. 86, pp. 2049-2069, Oct. 1998

[6] G. Woodward and B. Vucetic, "Adaptive detection of DS-CDMA," Proc. IEEE, vol. 86, pp. 1413-1434, July 1998.

[7] I. Ghauri and D. T. M. Slock, "Blind channel and linear MMSE receiver determination in DS-CDMA systems," in Proc. ICASSP'99, Mar. 1999, pp. 2699-2702.

[8] M. K. Tsatsanis and Z. Xu, "Performance analysis of minimum variance CDMA receivers," IEEE Trans. Signal Processing, vol. 46, pp. 3014-3022, Nov. 1998.

[9] X. Wang and H. V. Poor, "Blind adaptive multiuser detection in multipath CDMA channels based on subspace tracking," IEEE Trans. Signal Processing, vol. 46, pp. 3030-3044, Nov. 1998.

[10] H. Liu and M. D. Zoltowski, "Blind equalization in antenna array CDMA systems," IEEE Trans. Signal Processing, vol. 45, pp. 161-172, Jan 1997.

[11] T. F. Wong, T. M. Lok, J. S. Lehnert, and M. D. Zoltowski, “A linear receiver for direct-sequence spread-spectrum multiple-access systems with antenna arrays and blind adaptation," IEEE Trans. Inform. Theory, vol. 44, pp. 659-676, Mar. 1998.

[12] A. M. Sayeed, "Canonical multipath-Doppler coordinates in wireless communications," in 36th Annu. Allerton Conf. Communication, Control and Computing, Monticello, IL, 1998, pp. 536-545.

[13] A. M. Sayeed and B. Aazhang, "Joint multipath-Doppler diversity in mobile wireless communications," IEEE Trans. Commun., vol. 47, pp. 123-132, Jan. 1999.

[14] A. M. Sayeed, A. Sendonaris, and B. Aazhang, "Multiuser detection in fast fading multipath environments," IEEE J. Select. Areas Commun., vol. 16, pp. 1691-1701, Dec. 1998.

[15] P. A. Bello, "Characterization of randomly time-variant linear channels," IEEE Trans. Commun. Technol., vol. COM-11, pp. 360-393, Nov. 1963.

[16] T. Kailath, "Sampling models for linear time-variant filters," MIT Res. Lab. of Electron., Tech. Rep. 352, May 1959.

[17] G. H. Golub and C. F. Van Loan, Matrix Computations, 2nd ed. Baltimore, MD: Johns Hopkins Univ. Press, 1989.

[18] U. Madhow and M. L. Honig, "MMSE interference suppression for direct-sequence spread-spectrum CDMA," IEEE Trans. Commun., vol. 42, pp. 3178-3188, Dec. 1994

[19] X. Wang and H. V. Poor, "Blind multiuser detection: A subspace approach," IEEE Trans. Inform. Theory, vol. 44, pp. 677-690, Mar. 1998.

[20] H. V. Poor and S. Verdu, "Probability of error in MMSE multiuser detection," IEEE Trans. Inform. Theory, vol. 43, pp. 858-871, May 1997.
[21] S. Haykin, Adaptive Filter Theory, 3rd ed. Englewood Cliffs, NJ: Prentice-Hall, 1996.

[22] S. Verdu, "Optimum multi-user asymptotic efficiency," IEEE Trans. Commun., vol. 38, pp. 496-508, Apr. 1990.

[23] S. Bhashyam, A. M. Sayeed, and B. Aazhang, "Time-selective signaling and reception for communication over multipath fading channels," IEEE Trans. Commun., pp. 83-94, Jan. 2000.

[24] A. M. Sayeed, E. N. Onggosanusi, and B. D. Van Veen, "A canonical space-time characterization of mobile wireless channels," IEEE Commun. Lett., vol. 3, pp. 94-96, Apr. 1999.

[25] E. N. Onggosanusi, A. M. Sayeed, and B. D. Van Veen, "Canonical space-time coordinates for multiuser wireless communications," in Proc. 2nd IEEE Workshop on Signal Processing Advanced Wireless Communications (SPAWC), 1999.

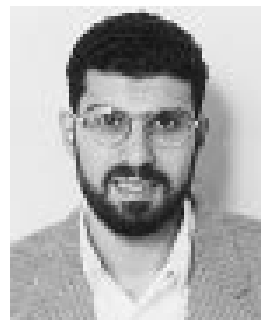

Tamer A. Kadous received the B.S. and M.S. degrees from the University of Alexandria, Alexandria, Egypt, in 1994 and 1997, respectively, and M.S. degree from the University of Wisconsin-Madison in 1999, all in electrical and computer engineering. He is currently working toward the Ph.D. degree in the Wireless Communication Group at the University of Wisconsin-Madison.

His research intrests include multiuser detection and OFDM communication systems. During the summer of 1999, he worked as an Intern at Nokia Research Center, Dallas, TX, where he received the 1999 Nokia Work Experience Award.

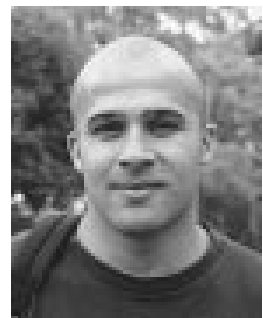

Akbar M. Sayeed (M'97) received the B.S. degree from the University of Wisconsin-Madison in 1991, and the M.S. and Ph.D. degrees from the University of Illinois at Urbana-Champaign, in 1993 and 1996, respectively, all in electrical and computer engineering.

While at the University of Illinois, he was a Research Assistant in the Coordinated Science Laboratory and was also the Schlumberger Fellow in Signal Processing from 1992 to 1995 . During 1996-1997, he was a Postdoctoral Fellow at Rice University, Houston, TX. Since August 1997, he has been with the University of Wisconsin-Madison, where he is currently an Assistant Professor in Electrical and Computer Engineering. His research interests include wireless communications, statistical signal processing, time frequency, and wavelet analysis.

Dr. Sayeed is currently serving as an Associate Editor for the IEEE SIGNAL PROCESSING LETTERS. In 1999, he received the National Science Foundation CAREER Award. 